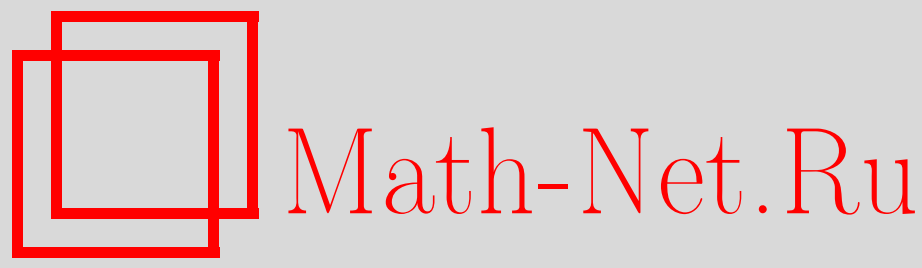

В. В. Жаринов, Анализ в дифференциальных алгебрах и модулях, ТМФ, 2018, том 196, номер 1, 3-21

DOI: https://doi.org/10.4213/tmf9566

Использование Общероссийского математического портала Math-Net.Ru подразумевает, что вы прочитали и согласны с пользовательским соглашением http: //www.mathnet.ru/rus/agreement

Параметры загрузки:

IP: 35.173 .219 .149

26 апреля 2023 г., 13:57:46

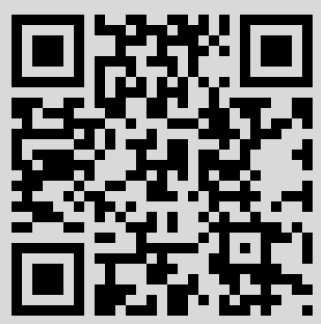




\title{
АНАЛИЗ В ДИФФЕРЕНЦИАЛЬНЫХ АЛГЕБРАХ И МОДУЛЯХ
}

\begin{abstract}
Предлагается краткое введение в математические методы и технику дифференциальных алгебр и модулей, адаптированную к задачам математической и теоретической физики.
\end{abstract}

Ключевые слова: алгебра, дифференциальная алгебра, модуль, дифференциальный модуль, мультипликатор, дифференцирование, комплекс де Рама, спектральная последовательность, вариационный бикомплекс.

DOI: https://doi.org/10.4213/tmf9566

\section{1. ВВЕДЕНИЕ}

Дифференциальные алгебры (см., например, [1]-[3]) широко известны и применяются в алгебре и топологии, тогда как их приложения в математической физике гораздо менее признанны (хотя и неявно используются, особенно в уравнениях в частных производных).

Здесь мы предлагаем короткое введение в математические методы и технику дифференциальных алгебр и модулей, адаптированную к задачам математической и теоретической физики. Изложение основано на личном опыте автора, книгах [1]--[7] и работах [8]-[13].

Мы пользуемся обозначениями, соглашениями и результатами статьи [14]. В частности:

- $\mathbb{F}=\mathbb{R}, \mathbb{C}$;

- $\mathbb{N}=\{1,2,3, \ldots\} \subset \mathbb{Z}_{+}=\{0,1,2, \ldots\} \subset \mathbb{Z}=\{0, \pm 1, \pm 2, \ldots\}$.

Мы также пользуемся следующими обозначениями теории гомологий (см., например, [4]):

- $\operatorname{Hom}\left(\mathcal{S} ; \mathcal{S}^{\prime}\right)$ есть множество всех отображений множества $\mathcal{S}$ в множество $\mathcal{S}^{\prime} ;$

Исследование выполнено за счет гранта Российского научного фонда (проект № 14-50-00005).

${ }^{*}$ Математический институт им. В. А. Стеклова Российской академии наук, Москва, Россия. E-mail: zharinov@mi.ras.ru 
- $\operatorname{Hom}_{\mathbb{F}}\left(\mathcal{L} ; \mathcal{L}^{\prime}\right)$ есть линейное пространство всех линейных отображений линейного пространства $\mathcal{L}$ в линейное пространство $\mathcal{L}^{\prime} ;$

- $\operatorname{Hom}_{\mathcal{A}}\left(\mathcal{M} ; \mathcal{M}^{\prime}\right)$ есть $\mathcal{A}$-модуль всех $\mathcal{A}$-линейных отображений $\mathcal{A}$-модуля $\mathcal{M}$ в $\mathcal{A}$-модуль $\mathcal{M}^{\prime}$, где $\mathcal{A}$ есть ассоциативная коммутативная алгебра;

- $\operatorname{Hom}_{\mathrm{alg}}\left(\mathcal{A} ; \mathcal{A}^{\prime}\right)$ есть множество всех морфизмов категории алгебр алгебры $\mathcal{A}$ в алгебру $\mathcal{A}^{\prime}$, где $\mathcal{A}, \mathcal{A}^{\prime}$ - ассоциативные коммутативные алгебры;

- $\operatorname{Hom}_{\text {Lie }}\left(\mathfrak{A} ; \mathfrak{A}^{\prime}\right)$ есть множество всех морфизмов категории алгебр Ли алгебры Ли $\mathfrak{A}$ в алгебру Ли $\mathfrak{A}^{\prime}$.

Напомним, что множество $\mathfrak{A}$ называется $\mathcal{A}$-алгеброй Лu, если на ней заданы две структуры:

- структура алгебры Ли со скобкой Ли $[\cdot, \cdot]$;

- структура $\mathcal{A}$-модуля, где $\mathcal{A}$ - ассоциативная коммутативная алгебра;

и эти структуры связаны условием согласования

- $[X, f \cdot Y]=X f \cdot Y+f \cdot[X, Y]$ для всех $X, Y \in \mathfrak{A}$ и $f \in \mathcal{A}$.

Обозначим через $\operatorname{Hom}_{\text {Lie } \cap \mathcal{A}}\left(\mathfrak{A} ; \mathfrak{A}^{\prime}\right)=\operatorname{Hom}_{\text {Lie }}\left(\mathfrak{A} ; \mathfrak{A}^{\prime}\right) \cap \operatorname{Hom}_{\mathcal{A}}\left(\mathfrak{A} ; \mathfrak{A}^{\prime}\right)$ множество всех морфизмов категории $\mathcal{A}$-алгебр Ли $\mathcal{A}$-алгебры Ли $\mathfrak{A}$ в $\mathcal{A}$-алгебру Ли $\mathfrak{A}^{\prime}$.

Все линейные операции выполняются над числовым полем $\mathbb{F}$. Как правило, предполагается суммирование по повторяющимся верхним и нижним индексам. Если соответствующее множество индексов бесконечное, мы предполагаем, что суммирование корректно определено. Если изучаемые объекты обладают естественными топологиями, мы предполагаем, что соответствующие отображения непрерывны. Например, если $\mathcal{S}$ и $\mathcal{S}^{\prime}$ суть топологические пространства, то $\operatorname{Hom}\left(\mathcal{S} ; \mathcal{S}^{\prime}\right)$ есть множество всех непрерывных отображений пространства $\mathcal{S}$ в пространство $\mathcal{S}^{\prime}$.

Мы используем терминологию, которая принята в алгебро-геометрическом подходе к уравнениям в частных производных, поскольку они являются основным объектом приложения развиваемой ниже техники.

\section{2. ДИФФЕРЕНЦИАЛЬНЫЕ АЛГЕБРЫ}

В этом разделе (подробнее см. [11], [14]) используются следующие обозначения:

- $\mathcal{A}$ - ассоциативная коммутативная алгебра;

- $\mathfrak{M}=\mathfrak{M}(\mathcal{A})=\operatorname{End}_{\mathcal{A}}(\mathcal{A})$ - унитальная ассоциативная алгебра всех мультипликаторов алгебры $\mathcal{A}$;

- $\mathfrak{D}=\mathfrak{D}(\mathcal{A})$ есть $\mathfrak{M}$-алгебра Ли всех дифберенцирований алгебры $\mathcal{A}$.

ОПРЕДЕЛЕНИЕ 1. Дифберенциальная алгебра есть пара $(\mathcal{A}, \mathcal{D})$, где $\mathcal{D}=\mathcal{D}(\mathcal{A})$ есть фиксированная подалгебра (картановская подалгебра) $\mathfrak{M}$-алгебры Ли $\mathfrak{D}=\mathfrak{D}(\mathcal{A})$.

ОПРЕДЕЛЕНИЕ 2. Пара $(F, \varphi)$, где отображение $F \in \operatorname{Hom}_{\mathrm{alg}}(\mathcal{A} ; \mathcal{B})$, а отображение $\varphi \in \operatorname{Hom}_{\text {Lie } \cap \mathfrak{M}}(\mathcal{D}(\mathcal{A}) ; \mathcal{D}(\mathcal{B}))$, называется морфизмом дифференциальной алгебры $(\mathcal{A}, \mathcal{D}(\mathcal{A}))$ в дифференциальную алгебру $(\mathcal{B}, \mathcal{D}(\mathcal{B}))$, если $F(X f)=(\varphi X)(F f)$ для всех $f \in \mathcal{A}, X \in \mathcal{D}(\mathcal{A})$. В этом случае мы будем писать $(F, \varphi):(\mathcal{A}, \mathcal{D}(\mathcal{A})) \rightarrow(\mathcal{B}, \mathcal{D}(\mathcal{B}))$.

Пусть $(\mathcal{A}, \mathcal{D})$ есть дифференциальная алгебра.

ОПредЕлЕниЕ 3. Подалгебра $\mathcal{B}$ (идеал $\mathcal{I}$ ) алгебры $\mathcal{A}$ называется дифференииальной (дифференииальным), если $\mathcal{D B} \subset \mathcal{B}$, т. е. $X f \in \mathcal{B}$ для всех $X \in \mathcal{D}$ и $f \in \mathcal{B}$ $(\mathcal{D} \mathcal{I} \subset \mathcal{I})$. В этом случае пара $(\mathcal{B}, \mathcal{D})$ (пара $(\mathcal{I}, \mathcal{D}))$ есть дифференциальная алгебра. 
В частности, если $(\mathcal{I}, \mathcal{D})$ есть дифференциальный идеал дифференциальной алгебры $(\mathcal{A}, \mathcal{D})$, то определена дифференциальная фактор-алгебра $(\mathcal{A}, \mathcal{D})$ правилом $\mathcal{A}=\mathcal{A} / \mathcal{I}, \mathcal{D}=\{\mathbf{X}=[X] \in \mathfrak{D}(\mathcal{A}) \mid X \in \mathcal{D}\},[X][f]=[X f]$ для всех $f \in \mathcal{A}$, где $[f]=f+\mathcal{I},[X f]=X f+\mathcal{I} \in \mathcal{A}$.

ПрЕДЛОЖЕНИЕ 1. Для всякого морфизма $(F, \varphi):(\mathcal{A}, \mathcal{D}(\mathcal{A})) \rightarrow(\mathcal{B}, \mathcal{D}(\mathcal{B}))$ ядро $\operatorname{Ker} F$ есть дифференииальный идеал алгебры $(\mathcal{A}, \mathcal{D}(\mathcal{A}))$, тогда как образ $\operatorname{Im} F$ является дифференииальной подалгеброй алгебры $(\mathcal{B}, \mathcal{D}(\mathcal{B}))$, если дополнительно отображение $\varphi: \mathcal{D}(\mathcal{A}) \rightarrow \mathcal{D}(\mathcal{B})$ сюргективно.

ОПреДЕЛЕНИЕ 4. Элемент $f \in \mathcal{A}$ называется $\mathcal{D}$-постоянным, если $\mathcal{D} f=0$, т. е. $X f=0$ для всех $X \in \mathcal{D}$.

Пусть $\mathcal{A}_{\mathcal{D}}$ есть множество всех $\mathcal{D}$-постоянных элементов алгебры $\mathcal{A}$. Очевидно, $\mathcal{A}_{\mathcal{D}}$ есть подалгебра алгебры $\mathcal{A}$.

ПрЕДЛОЖенИЕ 2. Для всякого морфизма $(F, \varphi):(\mathcal{A}, \mathcal{D}(\mathcal{A})) \rightarrow(\mathcal{B}, \mathcal{D}(\mathcal{B}))$, где отображение $\varphi$ сюрбективно, сужение $\left.F\right|_{\mathcal{A}_{\mathcal{D}}}: \mathcal{A}_{\mathcal{D}} \rightarrow \mathcal{B}_{\mathcal{D}}$.

ОПРЕДЕЛЕниЕ 5. Мультипликатор $R \in \mathfrak{M}$ называется $\mathcal{D}$-постоянным, если $\mathcal{D} R=$ $[\mathcal{D}, R]=0$, т. е. $[X, R]=0$ для всех $X \in \mathcal{D}$.

Пусть $\mathfrak{M}_{\mathcal{D}}$ есть множество всех $\mathcal{D}$-постоянных мультипликаторов алгебры $\mathfrak{M}$. Очевидно,

- $\mathfrak{M}_{\mathcal{D}}$ есть унитальная подалгебра алгебры $\mathfrak{M}$;

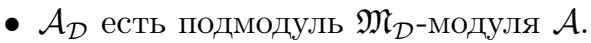

ОпределЕниЕ 6. Дифференцирование $X \in \mathfrak{D}$ называется дифференцированием Ли-Беклунда, если $[\mathcal{D}, X] \subset \mathcal{D}$, т. е. $[Y, X] \in \mathcal{D}$ для всех $Y \in \mathcal{D}$.

Пусть $\mathfrak{D}_{\mathcal{D}}=\mathfrak{D}_{\mathcal{D}}(\mathcal{A})$ есть множество всех дифференцирований Ли-Беклунда дифференциальной алгебры $(\mathcal{A}, \mathcal{D})$. Очевидно,

- $\mathfrak{D}_{\mathcal{D}}$ есть подалгебра $\mathfrak{M}_{\mathcal{D}}$-алгебры Ли $\mathfrak{D}$.

Предложение 3. Определена возрастающая фильтрация

$$
\mathcal{D}=\mathfrak{D}_{\mathcal{D}}^{(-1)} \subset \mathfrak{D}_{\mathcal{D}}=\mathfrak{D}_{\mathcal{D}}^{(0)} \subset \cdots \subset \mathfrak{D}_{\mathcal{D}}^{(q)} \subset \mathfrak{D}_{\mathcal{D}}^{(q+1)} \subset \cdots
$$

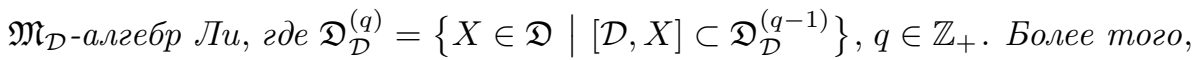

- $\mathcal{D}$ есть идеал $\mathfrak{M}_{\mathcal{D}}$-алгебры Ли $\mathfrak{D}_{\mathcal{D}}$;

- $\left[\mathfrak{D}_{\mathcal{D}}^{(p)}, \mathfrak{D}_{\mathcal{D}}^{(q)}\right] \subset \mathfrak{D}_{\mathcal{D}}^{(p+q)}$ для всех $p, q \in \mathbb{Z}_{+}$.

Общее определение фильтрации можно найти в книгах [4], [5]. Отметим, что мы не утверждаем, что $\bigcup_{q \in \mathbb{Z}_{+}} \mathfrak{D}_{\mathcal{D}}^{(q)}=\mathfrak{D}$ или $\lim _{q \rightarrow \infty} \mathfrak{D}_{\mathcal{D}}^{(q)}=\mathfrak{D}$ в каком-либо смысле.

ОПРеДЕЛЕниЕ 7. Дифференциальная алгебра $(\mathcal{A}, \mathcal{D})$ называется регулярной, если выполняются следующие условия:

- $\mathfrak{M}$-алгебра Ли $\mathfrak{D}$ разбита на вертикальную и горизонтальную подалгебры, $\mathfrak{D}=\mathfrak{D}_{V} \oplus_{\mathfrak{M}} \mathfrak{D}_{H}$

- вертикальная подалгебра $\mathfrak{D}_{V}=\mathfrak{D}_{V}(\mathcal{A})$ имеет $\mathfrak{M}$-базис $\partial=\left\{\partial_{a} \in \mathfrak{D}_{V} \mid a \in \mathbf{a}\right\}$, где $\mathbf{a}$ - множество индексов, $\left[\partial_{a}, \partial_{b}\right]=0, a, b \in \mathbf{a}$; 
- горизонтальная (картановская) подалгебра $\mathfrak{D}_{H}=\mathfrak{D}_{H}(\mathcal{A})=\mathcal{D}$ имеет $\mathfrak{M}$-базис $D=\left\{D_{\mu} \in \mathfrak{D}_{H} \mid \mu \in \mathbf{m}\right\}, \mathbf{m}=\{1, \ldots, m\}, m \in \mathbb{N},\left[D_{\mu}, D_{\nu}\right]=0, \mu, \nu \in \mathbf{m} ;$

- коммутаторы $\left[D_{\mu}, \partial_{a}\right]=\Gamma_{\mu a}^{b} \partial_{b} \in \mathfrak{D}_{V}, \mu \in \mathbf{m}, a, b \in \mathbf{a}$, коэффициенты $\Gamma_{\mu b}^{a} \in \mathfrak{M}$, в частности, $\left[D_{\mu}, X\right]=\nabla_{\mu} X=\left(\nabla_{\mu} X\right)^{a} \partial_{a} \in \mathfrak{D}_{V}$ для всех $X=X^{a} \partial_{a} \in \mathfrak{D}_{V}$, $X^{a} \in \mathfrak{M}$, где $\left(\nabla_{\mu} X\right)^{a}=D_{\mu} X^{a}+\Gamma_{\mu b}^{a} X^{b}$.

Пусть $\left(\mathcal{A}, \mathfrak{D}_{H}\right)$ есть регулярная дифференциальная алгебра.

ПРЕДЛОЖЕНИЕ 4. Коммутатор

$$
\left[\nabla_{\mu}, \nabla_{\nu}\right]_{b}^{a} X^{b}=\left(\left(D_{\mu} \Gamma_{\nu b}^{a}-D_{\nu} \Gamma_{\mu b}^{a}\right)+\left(\Gamma_{\mu c}^{a} \Gamma_{\nu b}^{c}-\Gamma_{\nu c}^{a} \Gamma_{\mu b}^{c}\right)\right) X^{b}, \quad a \in \mathbf{a}
$$

или, в матричных обозначениях, $\left[\nabla_{\mu}, \nabla_{\nu}\right]=F_{\mu \nu}$, где

- ковариантная производная $\nabla=\left(\nabla_{\mu}\right) \in \operatorname{Hom}_{\mathbb{F}}\left(\mathfrak{M}^{\mathbf{a}} ; \mathfrak{M}_{\mathbf{m}}^{\mathbf{a}}\right)$;

- связность $\Gamma=\left(\Gamma_{\mu}\right)$ с компонентами $\Gamma_{\mu}=\left(\Gamma_{\mu b}^{a}\right) \in \mathfrak{M}_{\mathbf{a}}^{\mathbf{a}}$;

- кривизна $F=\left(F_{\mu \nu}\right)$ с компонентами $F_{\mu \nu} \in \mathfrak{M}_{\mathbf{a}}^{\mathbf{a}}$;

- $F_{\mu \nu}=D_{\mu} \Gamma_{\nu}-D_{\nu} \Gamma_{\mu}+\left[\Gamma_{\mu}, \Gamma_{\nu}\right], \mu, \nu \in \mathbf{m}$.

\section{ПримеР 1. Пусть}

- $\mathrm{X}=\mathbb{R}^{\mathbf{m}}=\left\{x=\left(x^{\mu}\right) \mid x^{\mu} \in \mathbb{R}, \mu \in \mathbf{m}\right\}$ есть пространство независимых переменных;

- $\mathbf{U}=\mathbb{R}^{\mathbf{a}}=\left\{\mathbf{u}=\left(u^{a}\right) \mid u^{a} \in \mathbb{R}, a \in \mathbf{a}\right\}$ есть пространство дифференииальных переменных, a - бесконечное множество индексов;

- $\mathcal{A}=\mathcal{C}_{\text {fin }}^{\infty}(\mathrm{XU})$ есть унитальная ассоциативная коммутативная алгебра $\mathbb{F}$-значных гладких функций, зависящих от конечного числа аргументов $x^{\mu}, u^{a}$, где $\mathrm{XU}=\mathrm{X} \times \mathbf{U}$.

Разобьем $\mathcal{A}$-алгебру Ли $\mathfrak{D}=\mathfrak{D}(\mathcal{A})$ на вертикальную и горизонтальную части $\mathfrak{D}=$ $\mathfrak{D}_{\mathrm{V}} \oplus_{\mathcal{A}} \mathfrak{D}_{\mathrm{H}}$ следующим образом:

- $\mathfrak{D}_{\mathrm{V}}$ имеет $\mathcal{A}$-базис $\partial=\left\{\partial_{u^{a}} \mid a \in \mathbf{a}\right\}$;

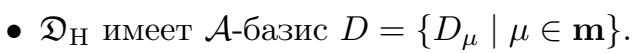

Здесь $D_{\mu}=\partial_{x^{\mu}}+h_{\mu}^{a} \cdot \partial_{u^{a}}$, а $\partial_{u^{a}}, \partial_{x^{\mu}}$ суть частные производные. Для выполнения условия $\left[D_{\mu}, D_{\nu}\right]=0$ мы предположим, что коэффициенты $h_{\mu}^{a} \in \mathcal{A}$ удовлетворяют равенствам $D_{\mu} h_{\nu}^{a}-D_{\nu} h_{\mu}^{a}=0$ для всех $a \in \mathbf{a}, \mu, \nu \in \mathbf{m}$.

В этом случае связность $\Gamma=\left(\Gamma_{\mu}\right)$ имеет компоненты $\Gamma_{\mu b}^{a}=-\partial_{u^{b}} h_{\mu}^{a}$, а кривизна $F=\left(F_{\mu \nu}\right)=0$.

С геометрической точки зрения множество $D=\left\{D_{\mu} \mid \mu \in \mathbf{m}\right\}$ определяет инволютивное (в смысле Фробениуса) распределение на пространстве XU. Каждая функция $\phi=\left(\phi^{a}(x)\right) \in \mathcal{C}^{\infty}(\mathrm{X} ; \mathbf{U})$ задает $m$-мерное подмногообразие $\Phi=\{\mathbf{u}=\phi(x)\}$ в XU. Это подмногообразие будет интегральным многообразием распределения $D$, если $\left.\left(D_{\mu}\left(u^{a}-\phi^{a}(x)\right)\right)\right|_{\mathbf{u}=\phi(x)}=0$ для всех $\mu \in \mathbf{m}, a \in \mathbf{a}$, т. е. если функция $\phi(x)$ является решением определяющей системы: $\partial_{x^{\mu}} \phi^{a}(x)-h_{\mu}^{a}(x, \phi(x)), \mu \in \mathbf{m}, a \in \mathbf{A}$. Эта система имеет условие интегрирования $\partial_{x^{\mu}} h_{\nu}^{a}\left(x, \phi(x)=\partial_{x^{\nu}} h_{\mu}^{a}(x, \phi(x)), \mu, \nu \in \mathbf{m}\right.$, $a \in \mathbf{a}$, для любого решения $\phi(x)$ системы. Последнее условие выполнено в силу 
предположения $D_{\mu} h_{\nu}^{a}-D_{\nu} h_{\mu}^{a}=0$ и правила дифференцирования сложной функции

$$
\partial_{x^{\mu}} f(x, \phi(x))=\left.\left(D_{\mu} f(x, \mathbf{u})\right)\right|_{\mathbf{u}=\phi(x)}
$$

для всех $\mu \in \mathbf{m}, f \in \mathcal{A}$ и любого решения $\phi(x)$ определяющей системы.

Пусть снова $\left(\mathcal{A}, \mathfrak{D}_{\mathrm{H}}\right)$ есть регулярная дифференциальная алгебра.

ПРЕДЛОЖЕНИЕ 5. Определена возрастающая филътрачия

$$
0 \subset \mathcal{A}_{\mathrm{H}}^{(0)}=\mathcal{A}_{\mathcal{D}} \subset \mathcal{A}_{\mathrm{H}}^{(1)} \subset \cdots \subset \mathcal{A}_{\mathrm{H}}^{(q)} \subset \mathcal{A}_{\mathrm{H}}^{(q+1)} \subset \cdots
$$

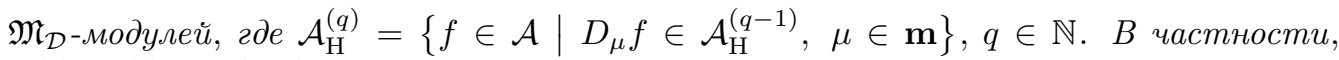
$\mathcal{A}_{\mathrm{H}}^{(p)} \cdot \mathcal{A}_{\mathrm{H}}^{(q)} \subset \mathcal{A}_{\mathrm{H}}^{(p+q)}$ для всех $p, q \in \mathbb{Z}_{+}$.

ПредлОЖЕНИЕ 6. Определена возрастающая фильтращия

$$
0 \subset \mathfrak{M}_{\mathrm{H}}^{(0)}=\mathfrak{M}_{\mathcal{D}} \subset \mathfrak{M}_{\mathrm{H}}^{(1)} \subset \cdots \subset \mathfrak{M}_{\mathrm{H}}^{(q)} \subset \mathfrak{M}_{\mathrm{H}}^{(q+1)} \subset \cdots
$$

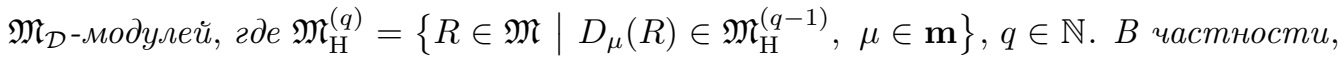
$\mathfrak{M}_{\mathrm{H}}^{(p)} \circ \mathfrak{M}_{\mathrm{H}}^{(q)} \subset \mathfrak{M}_{\mathrm{H}}^{(p+q)}$ для всех $p, q \in \mathbb{Z}_{+}$.

ПрЕДЛОЖЕниЕ 7. Определена возрастающая фильтрачия

$$
0=\mathcal{E}^{(-1)} \subset \mathcal{E}=\mathcal{E}^{(0)} \subset \cdots \subset \mathcal{E}^{(q)} \subset \mathcal{E}^{(q+1)} \subset \cdots
$$

$\mathfrak{M}_{\mathcal{D}}$-алгебр Ли, где $\mathcal{E}^{(q)}=\left\{X \in \mathfrak{D}_{\mathrm{V}} \mid\left[D_{\mu}, X\right] \in \mathcal{E}^{(q-1)}, \mu \in \mathbf{m}\right\}, q \in \mathbb{N}$. Более того,

- $\left[\mathcal{E}^{(p)}, \mathcal{E}^{(q)}\right] \subset \mathcal{E}^{(p+q)}, p, q \in \mathbb{Z}_{+}$;

- $\mathfrak{D}_{\mathcal{D}}^{(q)}=\mathcal{E}^{(q)} \oplus_{\mathfrak{M}_{\mathcal{D}}} \mathcal{D}, q \in \mathbb{Z}_{+}$.

\section{3. ДИФФЕРЕНЦИАЛЬНЫЕ МОДУЛИ}

Пусть $(\mathcal{A}, \mathcal{D})$ - дифференциальная алгебра, $\mathcal{M}-\mathcal{A}$-модуль, $\mathfrak{M}=\mathfrak{M}(\mathcal{M})$ - алгебра всех мультипликаторов $\mathcal{A}$-модуля $\mathcal{M}$, и $\mathfrak{D}=\mathfrak{D}(\mathcal{M})-\mathfrak{M}$-алгебра Ли всех дифференцирований $\mathcal{A}$-модуля $\mathcal{M}$.

ОПРЕДЕЛЕНИЕ 8. Дифференциальный модуль над дифференциальной алгеброй $(\mathcal{A}, \mathcal{D})$ (кратко, $(\mathcal{A}, \mathcal{D})$-модуль) есть тройка $(\mathcal{M}, \kappa, \mathcal{D})$, где

- $\mathcal{M}$ есть $\mathcal{A}$-модуль;

- отображение $\kappa \in \operatorname{Hom}_{\operatorname{Lie} \cap \mathcal{A}}(\mathfrak{D} ; \mathfrak{D})$, в частности $\kappa[X, Y]=[\kappa X, \kappa Y]$ для всех $X, Y \in \mathfrak{D}$

- $\mathcal{D}$ есть картановская подалгебра $\mathfrak{M}$-алгебры Ли $\mathfrak{D}$;

- условие согласования $\left.\kappa\right|_{\mathcal{D}}: \mathcal{D} \rightarrow \mathcal{D}$.

Пусть $(\mathcal{M}, \kappa, \mathcal{D})$ есть дифференциальный модуль.

ОПРЕДЕЛЕНИЕ 9. Подмодуль $\mathcal{N} \mathcal{A}$-модуля $\mathcal{M}$ называется дифференииалъным, если $\mathcal{D N} \subset \mathcal{N}$. В этом случае пара $(\mathcal{N}, \mathcal{D})$ есть дифференциальный модуль.

ОПРеДЕЛЕНИЕ 10. Элемент $M \in \mathcal{M}$ называется $\mathcal{D}$-постоянным, если $\mathcal{D} M=0$.

Пусть $\mathcal{M}_{\mathcal{D}}$ есть множество всех $\mathcal{D}$-постоянных элементов $\mathcal{A}$-модуля $\mathcal{M}$. 
ОПРЕДЕЛЕНИЕ 11. Мультипликатор $\mathbf{R} \in \mathfrak{M}$ называется $\mathcal{D}$-nостоянным, если $\mathcal{D}(\mathbf{R})=[\mathcal{D}, \mathbf{R}]=0$.

Пусть $\mathfrak{M}_{\mathcal{D}}$ есть множество всех $\mathcal{D}$-постоянных элементов алгебры $\mathfrak{M}$. Очевидно,

- $\mathfrak{M}_{\mathcal{D}}$ есть унитальная подалгебра алгебры $\mathfrak{M}$;

- $\mathcal{M}_{\mathcal{D}}$ есть подмодуль $\mathfrak{M}_{\mathcal{D}}$-модуля $\mathcal{M}$.

ОПРЕДЕЛЕНИЕ 12. Дифференцирование $\mathbf{X} \in \mathfrak{D}$ называется дифферениированием Ли-Беклунда, если $[\mathcal{D}, \mathbf{X}] \subset \mathcal{D}$.

Пусть $\mathfrak{D}_{\mathcal{D}}$ есть множество всех дифференцирований Ли-Беклунда дифференциального модуля $(\mathcal{M}, \mathcal{D})$. Очевидно,

- $\mathfrak{D}_{\mathcal{D}}$ есть подалгебра $\mathfrak{M}_{\mathcal{D}}$-алгебры Ли $\mathfrak{D}$.

ПрЕДЛОЖЕНИЕ 8. Определена возрастающая фильтращия

$$
\mathcal{D}=\mathfrak{D}_{\mathcal{D}}^{(-1)} \subset \mathfrak{D}_{\mathcal{D}}=\mathfrak{D}_{\mathcal{D}}^{(0)} \subset \cdots \subset \mathfrak{D}_{\mathcal{D}}^{(q)} \subset \mathfrak{D}_{\mathcal{D}}^{(q+1)} \subset \cdots
$$

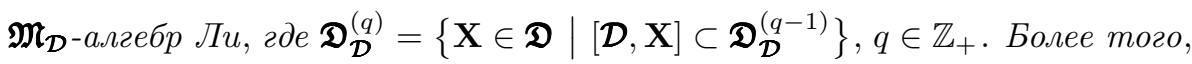

- $\mathcal{D}$ есть идеал $\mathfrak{M}_{\mathcal{D}}$-алгебри Ли $\mathfrak{D}_{\mathcal{D}}$;

- $\left[\mathfrak{D}_{\mathcal{D}}^{(p)}, \mathfrak{D}_{\mathcal{D}}^{(q)}\right] \subset \mathfrak{D}_{\mathcal{D}}^{(p+q)}$ для всех $p, q \in \mathbb{Z}_{+}$.

ОПРЕДЕЛЕНИЕ 13. Пусть $(\mathcal{A}, \mathcal{D})$ есть регулярная дифференциальная алгебра с вертикальным $\mathfrak{M - б а з и с о м ~} \partial=\left\{\partial_{a} \mid a \in \mathbf{a}\right\}$ и горизонтальным $\mathfrak{M - б а з и с о м ~} D=$ $\left\{D_{\mu} \mid \mu \in \mathbf{m}\right\}$. Дифференциальный модуль $(\mathcal{M}, \kappa, \mathcal{D})$ называется регулярным, если выполняются следующие условия:

- $\mathfrak{M}$-алгебра Ли $\mathfrak{D}$ разбита в вертикальную и горизонталъную подалгебры, $\mathfrak{D}=\mathfrak{D}_{\mathrm{V}} \oplus \mathfrak{M} \mathfrak{D}_{\mathrm{H}}$

- вертикальная подалгебра $\mathfrak{D}_{\mathrm{V}}$ имеет $\mathfrak{M}$-базис $\boldsymbol{\partial}=\left\{\boldsymbol{\partial}_{s}=\left(\nabla_{s}, \partial_{s}\right) \in \mathfrak{D}_{\mathrm{V}} \mid \partial_{s} \in\right.$ $\left.\partial, s \in \mathbf{a}_{\mathcal{M}}\right\},\left[\boldsymbol{\partial}_{s}, \partial_{t}\right]=0, s, t \in \mathbf{a}_{\mathcal{M}} ;$

- горизонтальная подалгебра $\mathfrak{D}_{\mathrm{H}}=\mathcal{D}$ имеет $\mathfrak{M}$-базис $\mathbf{D}=\left\{\mathbf{D}_{\sigma}=\left(\nabla_{\sigma}, D_{\sigma}\right) \in\right.$ $\left.\mathfrak{D}_{\mathrm{H}} \mid D_{\sigma} \in D, \sigma \in \mathbf{m}_{\mathcal{M}}\right\},\left[\mathbf{D}_{\sigma}, \mathbf{D}_{\tau}\right]=0, \sigma, \tau \in \mathbf{m}_{\mathcal{M}}$

- коммутаторы $\left[\mathbf{D}_{\sigma}, \partial_{s}\right]=\Gamma_{\sigma s}^{t} \partial_{t} \in \mathfrak{D}_{\mathrm{V}}, \Gamma_{\sigma s}^{t}=\left(\Delta_{\sigma s}^{t}, \Gamma_{\sigma s}^{t}\right) \in \mathfrak{M}$, в частности $\left[\mathbf{D}_{\sigma}, \mathbf{X}\right]=\boldsymbol{\nabla}_{\sigma} \mathbf{X}=\left(\boldsymbol{\nabla}_{\sigma} \mathbf{X}\right)^{s} \boldsymbol{\partial}_{s} \in \mathfrak{D}_{\mathrm{V}}$ для всех $\mathbf{X}=\mathbf{X}^{s} \boldsymbol{\partial}_{s} \in \mathfrak{D}_{\mathrm{V}}, \mathbf{X}^{s} \in \mathfrak{M}$, где $\left(\nabla_{\sigma} \mathbf{X}\right)^{s}=\mathbf{D}_{\sigma} \mathbf{X}^{s}+\Gamma_{\sigma t}^{s} \mathbf{X}^{t}$.

Пусть $\left(\mathcal{M}, \kappa, \mathfrak{D}_{\mathrm{H}}\right)$ есть регулярный дифференциальный модуль.

ПРЕДЛОЖЕНИЕ 9. Определена возрастающая фильтрация

$$
0 \subset \mathcal{M}_{\mathrm{H}}^{(0)}=\mathcal{M}_{\mathcal{D}} \subset \mathcal{M}_{\mathrm{H}}^{(1)} \subset \cdots \subset \mathcal{M}_{\mathrm{H}}^{(q)} \subset \mathcal{A}_{\mathrm{H}}^{(q+1)} \subset \cdots
$$

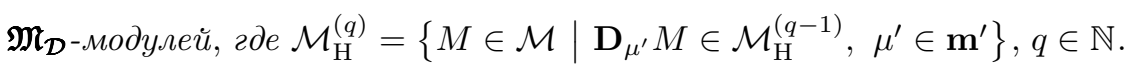


ПреДЛОЖЕНИЕ 10. Определена возрастающая фильтрация

$$
0 \subset \mathfrak{M}_{\mathrm{H}}^{(0)}=\mathfrak{M}_{\mathcal{D}} \subset \mathfrak{M}_{\mathrm{H}}^{(1)} \subset \cdots \subset \mathfrak{M}_{\mathrm{H}}^{(q)} \subset \mathfrak{M}_{\mathrm{H}}^{(q+1)} \subset \cdots
$$

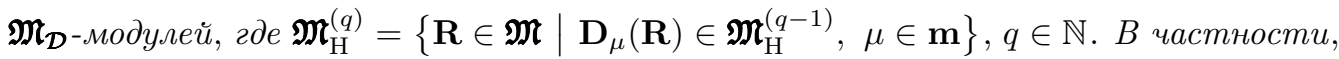
$\mathfrak{M}_{\mathrm{H}}^{(p)} \circ \mathfrak{M}_{\mathrm{H}}^{(q)} \subset \mathfrak{M}_{\mathrm{H}}^{(p+q)}$ для всех $p, q \in \mathbb{Z}_{+}$.

ПреДлОжЕНИЕ 11. Определена возрастающая фильтрачия

$$
0=\mathcal{E}^{(-1)} \subset \mathcal{E}=\mathcal{E}^{(0)} \subset \cdots \subset \mathcal{E}^{(q)} \subset \mathcal{E}^{(q+1)} \subset \cdots
$$

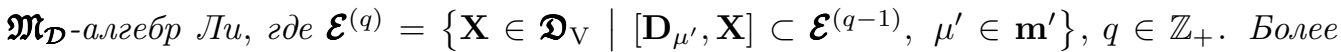
mого,

- $\left[\mathcal{E}^{(p)}, \mathcal{E}^{(q)}\right] \subset \mathcal{E}^{(p+q)}$ для всех $p, q \in \mathbb{Z}_{+}$;

- $\mathfrak{D}_{\mathcal{D}}^{(q)}=\mathcal{E}^{(q)} \oplus_{\mathfrak{M}_{\mathcal{D}}} \mathcal{D}$ для всех $q \in \mathbb{Z}_{+}$.

\section{4. СПЕКТРАЛЬНЫЕ ПОСЛЕДОВАТЕЛЬНОСТИ}

СоГЛАшЕНИЕ 1 . Пусть $\mathcal{U}=\mathcal{A}, \mathcal{M}$ и $\mathcal{V}=\mathcal{A}, \mathcal{K}$, где

- $(\mathcal{A}, \mathcal{D})$ - унитальная ассоциативная коммутативная алгебра;

- $\left(\mathcal{M}, \kappa=\kappa_{\mathcal{M}}, \mathcal{D}\right)-(\mathcal{A}, \mathcal{D})$-модуль, $\mathcal{K}-\mathcal{A}$-модуль;

- $\kappa_{\mathcal{K} \mathcal{M}} \in \operatorname{Hom}_{\operatorname{Lie} \cap \mathcal{A}}(\mathfrak{D}(\mathcal{M}) ; \mathfrak{D}(\mathcal{K})), \kappa_{\mathcal{K}} \in \operatorname{Hom}_{\text {Lie } \cap \mathcal{A}}(\mathfrak{D}(\mathcal{A}) ; \mathfrak{D}(\mathcal{K}))$, $\kappa_{\mathcal{K}}=\kappa_{\mathcal{K} \mathcal{M}} \circ \kappa_{\mathcal{M}}$

СоГлАшЕНИЕ 2. Для упрощения обозначений ниже мы будем писать $\varkappa: \mathfrak{D}(\mathcal{U}) \rightarrow$ $\mathfrak{D}(\mathcal{V})$, где

$$
\begin{array}{ll}
\varkappa=\operatorname{id}_{\mathfrak{D}(\mathcal{A})}, \quad \text { когда } \mathcal{U}=\mathcal{V}=\mathcal{A}, & \varkappa=\kappa_{\mathcal{K}}, \quad \text { когда } \mathcal{U}=\mathcal{A}, \mathcal{V}=\mathcal{K}, \\
\varkappa=\Pi, \quad \text { когда } \mathcal{U}=\mathcal{M}, \mathcal{V}=\mathcal{A}, \quad \varkappa=\varkappa_{\mathcal{M}}, \quad \text { когда } \mathcal{U}=\mathcal{M}, \mathcal{V}=\mathcal{K},
\end{array}
$$

проекция П: $\mathfrak{D}(\mathcal{M}) \rightarrow \mathfrak{D}(\mathcal{A}), \mathbf{X}=\left(\nabla_{X}, X\right) \mapsto X$ (см. [14]).

ОПРЕДЕЛЕНИЕ 14. $\mathcal{A}$-модуль $\Omega(\mathcal{U} ; \mathcal{V})=\oplus_{r \in \mathbb{Z}} \Omega^{r}(\mathcal{U} ; \mathcal{V})$ дифференциальных форм над $\mathcal{U}$ с коэффициентами в $\mathcal{V}$ определяется правилом (см., например, [14])

$$
\Omega^{r}(\mathcal{U}, \mathcal{V})= \begin{cases}0, & r<0 \\ \mathcal{V}, & r=0 \\ \operatorname{Hom}_{\mathcal{A}}\left(\wedge_{\mathcal{A}}^{r} \mathfrak{D}(\mathcal{U}) ; \mathcal{V}\right), & r>0\end{cases}
$$

Множество $\Omega(\mathcal{U}, \mathcal{A})$ имеет структуру внешней $\mathcal{A}$-алгебры, а множество $\Omega(\mathcal{U}, \mathcal{K})$ имеет структуру внешнего $\Omega(\mathcal{U}, \mathcal{A})$-модуля. Более того, в общем случае $\Omega(\mathcal{U}, \mathcal{V})=$ $\Omega(\mathcal{U}, \mathcal{A}) \otimes_{\mathcal{A}} \mathcal{V}$

ПРЕДЛОЖЕНИЕ 12. Для всякого дифберенцирования $X \in \mathfrak{D}(\mathcal{U})$ справедливъ следующие утверждения:

- внутреннее произведение $i_{X} \in \operatorname{End}_{\mathcal{A}}(\Omega(\mathcal{U}, \mathcal{V}))$ определяется правилом свертки $i_{X} \omega\left(X_{1}, \ldots, X_{r-1}\right)=r \omega\left(X, X_{1}, \ldots, X_{r-1}\right)$ для всех $r \in \mathbb{Z}, \omega \in \Omega^{r}(\mathcal{U}, \mathcal{V})$, $X_{1}, \ldots, X_{r-1} \in \mathfrak{D}(\mathcal{U})$; 
- производная Ли $L_{X} \in \operatorname{End}_{\mathbb{F}}(\Omega(\mathcal{U}, \mathcal{V}))$ определяется правилом

$$
L_{X} \omega\left(X_{1}, \ldots, X_{r}\right)=(\varkappa X) \omega\left(X_{1}, \ldots, X_{r}\right)-\sum_{1 \leqslant s \leqslant r} \omega\left(X_{1}, \ldots\left[X, X_{s}\right] \ldots, X_{r}\right)
$$

для всех $r \in \mathbb{Z}, \omega \in \Omega^{r}(\mathcal{U}, \mathcal{V}), X_{1}, \ldots, X_{r} \in \mathfrak{D}(\mathcal{U})$;

- $i_{X}$ есть внешнее дифференцирование, а $L_{X}$ есть дифференцирование $\Omega(\mathcal{U}, \mathcal{A})$ модуля $\Omega(\mathcal{U}, \mathcal{V})$, m.e.

$$
\begin{aligned}
& i_{X}(\omega \wedge \chi)=\left(i_{X} \omega\right) \wedge \chi+(-1)^{r} \omega \wedge\left(i_{X} \chi\right), \\
& L_{X}(\omega \wedge \chi)=\left(L_{X} \omega\right) \wedge \chi+\omega \wedge\left(L_{X} \chi\right)
\end{aligned}
$$

для всех $\omega \in \Omega^{r}(\mathcal{U}, \mathcal{A}), r \in \mathbb{Z}_{+}, \chi \in \Omega(\mathcal{U}, \mathcal{V})$.

ДоКАЗАТЕЛЬСТво предложения 12 можно найти, например, в работе [14].

ОПРЕДЕЛЕНИЕ 15. Форма $\omega \in \Omega^{r}(\mathcal{U}, \mathcal{V})$ называется р-картановской формой, $0 \leqslant p \leqslant r$, если $\omega\left(X_{1}, \ldots, X_{r}\right)=0$, когда по крайней мере $r-p+1$ дифференцирований $X_{1}, \ldots, X_{r}$ картановские, т. е. принадлежат подалгебре $\mathcal{D}(\mathcal{U}) \subset \mathfrak{D}(\mathcal{U})$.

Пусть $\Omega_{p}^{r}(\mathcal{U}, \mathcal{V})$ есть $\mathcal{A}$-модуль всех $p$-картановских форм из $\Omega^{r}(\mathcal{U}, \mathcal{V})$.

ПРеДЛОЖениЕ 13. Определена убывающая фильтрация $\mathcal{A}$-модулей

$$
\Omega_{0}^{r}(\mathcal{U}, \mathcal{V})=\Omega^{r}(\mathcal{U}, \mathcal{V}) \supset \Omega_{1}^{r}(\mathcal{U}, \mathcal{V}) \supset \cdots \supset \Omega_{r}^{r}(\mathcal{U}, \mathcal{V}) \supset 0, \quad r \in \mathbb{Z} .
$$

Более того,

- $\Omega_{p}^{r}(\mathcal{U}, \mathcal{A}) \wedge_{\mathcal{A}} \Omega_{q}^{s}(\mathcal{U}, \mathcal{V}) \subset \Omega_{p+q}^{r+s}(\mathcal{U}, \mathcal{V})$ для всех возможных $p, q, r, s \in \mathbb{Z}$.

ПРЕДЛОЖЕНИЕ 14. Пусть $\omega \in \Omega_{p}^{r}(\mathcal{U}, \mathcal{V}), p, r \in \mathbb{Z}$, тогда

$$
i_{X} \omega \in\left\{\begin{array} { l l } 
{ \Omega _ { p - 1 } ^ { r - 1 } ( \mathcal { U } , \mathcal { V } ) , } & { X \in \mathfrak { D } ( \mathcal { U } ) , } \\
{ \Omega _ { p } ^ { r - 1 } ( \mathcal { U } , \mathcal { V } ) , } & { X \in \mathcal { D } ( \mathcal { U } ) , }
\end{array} \quad L _ { X } \omega \in \left\{\begin{array}{ll}
\Omega_{p-1}^{r}(\mathcal{U}, \mathcal{V}), & X \in \mathfrak{D}(\mathcal{U}), \\
\Omega_{p}^{r}(\mathcal{U}, \mathcal{V}), & X \in \mathfrak{D}_{\mathcal{D}}(\mathcal{U}) .
\end{array}\right.\right.
$$

Напомним (см., например, [14]), что формулой Картана определено отображение $d=d_{\varkappa} \in \operatorname{End}_{\mathbb{F}}(\Omega(\mathcal{U}, \mathcal{V}))$

$$
\begin{aligned}
d \omega\left(X_{0}, \ldots, X_{r}\right)= & \frac{1}{r+1}\left\{\sum_{0 \leqslant s \leqslant r}(-1)^{s}\left(\varkappa X_{s}\right) \omega\left(x_{0}, \ldots, \check{X}_{s}, \ldots, X_{r}\right)+\right. \\
& \left.+\sum_{0 \leqslant s<t \leqslant r}(-1)^{s+t} \omega\left(\left[X_{s}, X_{t}\right], X_{0}, \ldots, \check{X}_{s}, \ldots, \check{X}_{t}, \ldots, X_{r}\right)\right\}
\end{aligned}
$$

для всех $r \in \mathbb{Z}_{+}, \omega \in \Omega^{r}(\mathcal{U}, \mathcal{V}), X_{0}, \ldots, X_{r} \in \mathfrak{D}(\mathcal{U})$, где предполагается, что аргументы под знаком " 2 " пропущены. 
ПреДЛОЖЕНИЕ 15. Справедливы следующие утверждения:

- $d^{r}=\left.d\right|_{\Omega^{r}(\mathcal{U}, \mathcal{V})}: \Omega^{r}(\mathcal{U}, \mathcal{V}) \rightarrow \Omega^{r+1}(\mathcal{U}, \mathcal{V})$

- эндоморфизм d есть внешнее дифберенцирование внешней $\mathcal{A}$-алгебры $\Omega(\mathcal{U}, \mathcal{A})$ и внешнего $\Omega(\mathcal{U}, \mathcal{A})$-модуля $\Omega(\mathcal{U}, \mathcal{M})$;

- композииия $d \circ d=0$.

ДокАЗАТЕЛЬСтво предложения 15 можно найти в работе [14] (теорема 6).

В частности, определен комплекс де Рама $\left\{\Omega^{r}(\mathcal{U}, \mathcal{V}), d^{r} \mid r \in \mathbb{Z}\right\}$ с пространствами когомологий $H^{r}(\mathcal{U}, \mathcal{V})=\operatorname{Ker} d^{r} / \operatorname{Im} d^{r-1}, r \in \mathbb{Z}$.

ПреДЛОЖЕНИЕ 16. Для всякого дифферениирования $X \in \mathfrak{D}(\mathcal{U})$ справедлива "магическая" бормула Картана

$$
L_{X}=d \circ i_{X}+i_{X} \circ d
$$

В частности, $L_{X} \circ d=d \circ L_{X}$ для любого $X \in \mathfrak{D}(\mathcal{U})$.

ДокАЗАТЕЛЬСТво предложения 16 можно найти в работе [14] (теорема 7).

Фильтрация $\left\{\Omega_{p}^{r}(\mathcal{U}, \mathcal{V}) \mid p \in \mathbb{Z}\right\}, r \in \mathbb{Z}$, позволяет рафинировать комплекс де Рама до спектральной последовательности.

ПрЕДЛОЖЕНИЕ 17. Филътрачия $\left\{\Omega_{p}^{r}(\mathcal{U}, \mathcal{V})\right\}$ совместима с дифференииалом $d \in$ $\operatorname{End}_{\mathbb{F}}(\Omega(\mathcal{U}, \mathcal{V}))$, а именно:

$$
\left.d\right|_{\Omega_{p}^{r}(\mathcal{U}, \mathcal{V})}: \Omega_{p}^{r}(\mathcal{U}, \mathcal{V}) \rightarrow \Omega_{p}^{r+1}(\mathcal{U}, \mathcal{V}) \quad \text { для всех } \quad r, p \in \mathbb{Z}_{+} .
$$

ПрЕДЛОЖЕНИЕ 18. Определена спектралъная последователъность ${ }^{1)}\left\{\mathrm{E}_{r}^{p q}, d_{r}^{p q}\right.$ $p, q, r \in \mathbb{Z}\}$, где

- $\mathrm{E}_{r}^{p q}=\mathrm{Z}_{r}^{p q} /\left(\mathrm{B}_{r-1}^{p q}+\mathrm{Z}_{r-1}^{p+1, q-1}\right)$;

- $\mathrm{Z}_{r}^{p q}=\left\{\omega \in \Omega_{p}^{p+q}(\mathcal{U}, \mathcal{V}) \mid d \omega \in \Omega_{p+r}^{p+q+1}(\mathcal{U}, \mathcal{V})\right\}$;

- $\mathrm{B}_{r}^{p q}=\left\{\omega=d \chi \in \Omega_{p}^{p+q}(\mathcal{U}, \mathcal{V}) \mid \chi \in \Omega_{p-r}^{p+q-1}(\mathcal{U}, \mathcal{V})\right\}$;

- $d_{r}^{p q}: \mathrm{E}_{r}^{p q} \rightarrow \mathrm{E}_{r}^{p+r, q-r+1}, \quad d_{r}^{p q}[\omega]_{r}^{p q}=[d \omega]_{r}^{p+r, q-r+1}$, где $[\omega]_{r}^{p q}$ есть класс эквивалентности формы $\omega \in \mathrm{Z}_{r}^{p q}$ в факторпространcmвe $\mathrm{E}_{r}^{p q}$.

В частности, из предложения 18 вытекают следующие равенства:

- $\mathrm{E}_{r}^{p q}=\Omega_{p}^{p+q}(\mathcal{U}, \mathcal{V}) / \Omega_{p+1}^{p+q}(\mathcal{U}, \mathcal{V})$ для всех $r \leqslant 0, p, q \in \mathbb{Z}$;

- $\mathrm{E}_{r}^{p q}=0$ для всех $p<0, r, q \in \mathbb{Z}$ и всех $q<0, r, p \in \mathbb{Z}$;

- $\mathrm{E}_{r+1}^{p q}=\operatorname{Ker} d_{r}^{p q} / \operatorname{Im} d_{r}^{p-r, q+r-1}$ для всех $p, q, r \in \mathbb{Z}$.

ПредложЕниЕ 19. Определены пределъные члены спектралъной последовательности $\left\{\mathrm{E}_{r}^{p q}, d_{r}^{p q} \mid p, q, r \in \mathbb{Z}\right\}$, а именно:

- $\mathrm{E}_{\infty}^{p q}=\mathrm{Z}_{\infty}^{p q} /\left(\mathrm{B}_{\infty}^{p q}+\mathrm{Z}_{\infty}^{p+1, q-1}\right)$;

- $\mathrm{Z}_{\infty}^{p q}=\left\{\omega \in \Omega_{p}^{p+q}(\mathcal{U}, \mathcal{V}) \mid d \omega=0\right\}$;

- $\mathrm{B}_{\infty}^{p q}=\left\{\omega=d \chi \in \Omega_{p}^{p+q}(\mathcal{U}, \mathcal{V}) \mid \chi \in \Omega^{p+q-1}(\mathcal{U}, \mathcal{V})\right\}$.

\footnotetext{
1) Общее определение см., например, в [4], [5].
} 
ОПРЕДЕЛЕНИЕ 16. Фильтрация $\left\{\Omega_{p}^{r}(\mathcal{U}, \mathcal{V})\right\}$ называется картановской, если

$$
\Omega_{0}^{r}(\mathcal{U}, \mathcal{V})=\cdots=\Omega_{r-m}^{r}(\mathcal{U}, \mathcal{V}) \supset \Omega_{r-m+1}^{r}(\mathcal{U}, \mathcal{V}) \supset \cdots \supset \Omega_{r}^{r}(\mathcal{U}, \mathcal{V}) \supset 0
$$

для некоторого числа $m \in \mathbb{N}$. Это число называется картановской размерностью $\mathcal{A}$-алгебры Ли $\mathcal{D}(\mathcal{U})$.

ПрЕДЛОЖЕНИЕ 20. Пусть $\left\{\Omega_{p}^{r}(\mathcal{U}, \mathcal{V})\right\}$ есть картановская фильтрачия. Тогда для всех $p, q \in \mathbb{Z}$ выполняются предельные равенства

$$
\lim _{r \rightarrow \infty} \mathrm{E}_{r}^{p q}=\mathrm{E}_{\infty}^{p q}
$$

ДокАзАТЕЛЬство. Существование пределов следует из общих свойств спектральных последовательностей (подробнее см. [7]).

В приложениях, как правило, используются лишь отдельные члены спектральных последовательностей. Так, в алгебро-геометрическом подходе к уравнениям в частных производных возникает картановская спектральная последовательность $\left\{\mathrm{E}_{r}^{p g}, d_{r}^{p q} \mid p, r \in \mathbb{Z}_{+}, 0 \leqslant q \leqslant m\right\}$ ( $m$ есть картановская размерность, см. [8] и, например, [15]), и важную роль играют следующие члены:

- $\mathrm{E}_{1}^{0 m}$ есть пространство функционалов, элементы классов эквивалентности называются лагранжианами;

- $\mathrm{E}_{1}^{0, m-1}$ есть постранство законов сохранения, элементы классов эквивалентности называются сохраняющимися токами;

- $\mathrm{E}_{1}^{0, q}, 0 \leqslant q \leqslant m-2$, суть пространства законов сохранения низшего порядка;

- $\mathrm{E}_{1}^{p m}, p \in \mathbb{N}$, суть пространства функциональных р-форм;

- $d_{1}^{p m}: \mathrm{E}_{1}^{p m} \rightarrow \mathrm{E}_{1}^{p+1, m}, p \in \mathbb{Z}_{+}$, суть функциональные (вариационные) дифференицаль, дифференциал $\delta=d_{1}^{0 m}$ называется оператором Эйлера-Лагранжа.

ОПРЕДЕЛЕНИЕ 17. Фактор-алгебра Ли $\operatorname{Sym}_{\mathcal{D}} \mathcal{U}=\mathfrak{D}_{\mathcal{D}}(\mathcal{U}) / \mathcal{D}(\mathcal{U})$ называется алгеброй симметрий дифференциальной алгебры (дифференциального модуля) $\mathcal{U}$.

ПРЕДЛОЖЕНИЕ 21. Для всякой симметрии $[X]=X+\mathcal{D}(U) \in \operatorname{Sym}_{\mathcal{D}} \mathcal{U}, X \in$ $\mathfrak{D}_{\mathcal{D}}(\mathcal{U})$, определены следующие фактор-морбизмы:

- $\left(i_{[X]}\right)_{r}: \mathrm{E}_{r}^{p q} \rightarrow \mathrm{E}_{r}^{p-1, q}, \quad[\omega]_{r}^{p q} \mapsto\left[i_{X} \omega\right]_{r}^{p-1, q}$

- $\left(L_{[X]}\right)_{1}: \mathrm{E}_{1}^{p q} \rightarrow \mathrm{E}_{1}^{p q}, \quad[\omega]_{1}^{p q} \mapsto\left[L_{X} \omega\right]_{1}^{p q}$.

\section{5. ВАРИАЦИОННЫЕ БИКОМПЛЕКСЫ}

Мы сохраняем все обозначения и соглашения предыдущего раздела.

СоглАшениЕ 3. Будем предполагать, что дифференциальная алгебра $(\mathcal{A}, \mathcal{D})$ и дифференциальный модуль $\left(\mathcal{M}, \kappa=\kappa_{\mathcal{M}}, \mathcal{D}\right)$ регулярные. В этом случае картановские размерности суть $m_{\mathcal{A}}=\operatorname{dim}_{\mathcal{A}} \mathcal{D}, m_{\mathcal{M}}=\operatorname{dim}_{\mathcal{A}} \mathcal{D}$.

Разбиение алгебр $\mathfrak{D}(\mathcal{A})$ и $\mathfrak{D}(\mathcal{K})$ на вертикальную и горизонтальную части позволяет рафинировать спектральную последовательность до вариационного бикомплекса (подробную информацию о бикомплексах можно найти, например, в [4] или [16]). 
ОПРЕДЕЛЕНИЕ 18. $\mathcal{A}$-модули $\Omega^{p q}(\mathcal{U}, \mathcal{V}), p, q \in \mathbb{Z}$, задаются правилом

$$
\Omega^{p q}(\mathcal{U}, \mathcal{V})= \begin{cases}0, & p<0 \text { и или } q<0 \\ \mathcal{V}, & p=q=0 \\ \operatorname{Hom}_{\mathcal{A}}\left(\left(\wedge_{\mathcal{A}}^{p} \mathfrak{D}_{\mathrm{V}}(\mathcal{U})\right) \wedge_{\mathcal{A}}\left(\wedge_{\mathcal{A}}^{q} \mathfrak{D}_{\mathrm{H}}(\mathcal{U})\right) ; \mathcal{V}\right), & p+q>0\end{cases}
$$

В частности, $\Omega_{\mathrm{V}}^{p}(\mathcal{U} ; \mathcal{V})=\Omega^{p 0}(\mathcal{U} ; \mathcal{V}), \Omega_{\mathrm{H}}^{q}(\mathcal{U} ; \mathcal{V})=\Omega^{0 q}(\mathcal{U} ; \mathcal{V})$ суть $\mathcal{A - м о д у л и ~ в е р т и - ~}$ кальных и горизонтальных форм соответственно. Более того, в общем случае $\Omega^{p q}(\mathcal{U} ; \mathcal{V})=\Omega_{\mathrm{V}}^{p}(\mathcal{U} ; \mathcal{A}) \wedge_{\mathcal{A}} \Omega_{\mathrm{H}}^{q}(\mathcal{U} ; \mathcal{A}) \otimes_{\mathcal{A}} \mathcal{V}$

Заметим, что $\Omega_{\mathrm{H}}^{q}(\mathcal{U}, \mathcal{V})=0$ для всех $q>m_{\mathcal{U}}$.

Разбиение $\mathfrak{D}(\mathcal{U})=\mathfrak{D}_{\mathrm{V}}(\mathcal{U}) \oplus_{\mathcal{A}} \mathfrak{D}_{\mathrm{H}}(\mathcal{U})$ определяет проекцию

$$
\pi_{\mathrm{V}, \mathrm{H}}: \mathfrak{D}(\mathcal{U}) \rightarrow \mathfrak{D}_{\mathrm{V}, \mathrm{H}}(\mathcal{U}), \quad X=X_{\mathrm{V}}+X_{\mathrm{H}} \mapsto \pi_{\mathrm{V}, \mathrm{H}} X=X_{\mathrm{V}, \mathrm{H}}
$$

Следовательно, определены дуальные инъекции

$$
\pi_{\mathrm{V}, \mathrm{H}}^{*}: \Omega_{\mathrm{V}, \mathrm{H}}^{r}(\mathcal{U}, \mathcal{V}) \rightarrow \Omega^{r}(\mathcal{U}, \mathcal{V}), \quad \omega \mapsto \pi_{\mathrm{V}, \mathrm{H}}^{*} \omega=\omega \circ \pi_{\mathrm{V}, \mathrm{H}}
$$

где $\pi_{\mathrm{V}, \mathrm{H}}^{*} \omega\left(X_{1}, \ldots, X_{r}\right)=\omega\left(\pi_{\mathrm{V}, \mathrm{H}} X_{1}, \ldots, \pi_{\mathrm{V}, \mathrm{H}} X_{s}\right), X_{1}, \ldots, X_{r} \in \mathfrak{D}(\mathcal{U})$.

ПРЕДЛОЖЕНИЕ 22. Отождествление $\pi_{\mathrm{V}, \mathrm{H}}^{*} \omega=\omega$ приводит $к$ представлениям

$$
\Omega^{r}(\mathcal{U}, \mathcal{V})=\oplus_{s \in \mathbb{Z}} \Omega^{s, r-s}(\mathcal{U}, \mathcal{V}), \quad \Omega_{p}^{r}(\mathcal{U}, \mathcal{V})=\oplus_{s \geqslant p} \Omega^{s, r-s}(\mathcal{U}, \mathcal{V})
$$

В частности, $\mathcal{A}$-модуль $\Omega(\mathcal{U}, \mathcal{V})=\oplus_{p, q \in \mathbb{Z}} \Omega^{p q}(\mathcal{U}, \mathcal{V})$ биградуирован.

По соглашению 3 унитальная дифференциальная алгебра $(\mathcal{A}, \mathcal{D})$ регулярная, т. е. $\mathfrak{D}(\mathcal{A})=\mathfrak{D}_{\mathrm{V}}(\mathcal{A}) \oplus_{\mathcal{A}} \mathfrak{D}_{\mathrm{H}}(\mathcal{A})$, где вертикальная подалгебра $\mathfrak{D}_{\mathrm{V}}(\mathcal{A})$ имеет $\mathcal{A}$-базис $\partial=$ $\left\{\partial_{a} \mid a \in \mathbf{a}\right\},\left[\partial_{a}, \partial_{b}\right]=0$, а горизонтальная подалгебра $\mathfrak{D}_{\mathrm{H}}(\mathcal{A})=\mathcal{D}$ имеет $\mathcal{A}$-базис $D=\left\{D_{\mu} \mid \mu \in \mathbf{m}\right\},\left[D_{\mu}, D_{\nu}\right]=0$.

В этом случае дуальный $\mathcal{A}$-модуль $\mathfrak{D}_{\mathrm{V}}^{*}(\mathcal{A})=\operatorname{Hom}_{\mathcal{A}}\left(\mathfrak{D}_{\mathrm{V}}(\mathcal{A}) ; \mathcal{A}\right)=\Omega_{\mathrm{V}}^{1}(\mathcal{A}, \mathcal{A})$ имеет дуальный $\mathcal{A}$-базис $\rho=\left\{\rho^{a} \in \mathfrak{D}_{\mathrm{V}}^{*}(\mathcal{A}) \mid a \in \mathbf{a}\right\}, \rho^{a}\left(\partial_{b}\right)=\delta_{b}^{a}$. В частности, $\omega(X)=$ $\omega_{a} \rho^{a}\left(X^{b} \partial_{b}\right)=\omega_{a} X^{a}$ для всех $\omega \in \mathfrak{D}_{\mathrm{V}}^{*}(\mathcal{A}), X \in \mathfrak{D}_{\mathrm{V}}(\mathcal{A})$ и $\omega(X)=0$ для всех $\omega \in$ $\mathfrak{D}_{\mathrm{V}}^{*}(\mathcal{A}), X \in \mathfrak{D}_{\mathrm{H}}(\mathcal{A})$.

Дуальный $\mathcal{A}$-модуль $\mathfrak{D}_{\mathrm{H}}^{*}(\mathcal{A})=\operatorname{Hom}_{\mathcal{A}}\left(\mathfrak{D}_{\mathrm{H}}(\mathcal{A}) ; \mathcal{A}\right)=\Omega_{\mathrm{H}}^{1}(\mathcal{A}, \mathcal{A})$ имеет дуальный $\mathcal{A}$-базис $\vartheta=\left\{\vartheta^{\mu} \mid \mu \in \mathbf{m}\right\}, \vartheta^{\mu}\left(D_{\nu}\right)=\delta_{\nu}^{\mu}$. В частности, $\omega(X)=\omega_{\mu} \vartheta^{\mu}\left(X^{\nu} D_{\nu}\right)=\omega_{\mu} X^{\mu}$ для всех $\omega \in \mathfrak{D}_{\mathrm{H}}^{*}(\mathcal{A}), X \in \mathfrak{D}_{\mathrm{H}}(\mathcal{A})$ и $\omega(X)=0$ для любых $\omega \in \mathfrak{D}_{\mathrm{H}}(\mathcal{A}), X \in \mathfrak{D}_{\mathrm{V}}(\mathcal{A})$.

То же самое верно и для $(\mathcal{A}, \mathcal{D})$-модуля $\left(\mathcal{M}, \kappa=\kappa_{\mathcal{M}}, \mathcal{D}\right)$. Именно ${ }^{2)}, \mathfrak{D}(\mathcal{M})=$ $\mathfrak{D}_{\mathrm{V}}(\mathcal{M}) \oplus_{\mathcal{A}} \mathfrak{D}_{\mathrm{H}}(\mathcal{M})$, вертикальная подалгебра $\mathfrak{D}_{\mathrm{V}}(\mathcal{M})$ имеет $\mathcal{A}$-базис $\boldsymbol{\partial}=\left\{\boldsymbol{\partial}_{s}\right.$ $\left.s \in \mathbf{a}_{\mathcal{M}}\right\},\left[\boldsymbol{\partial}_{s}, \boldsymbol{\partial}_{t}\right]=0$, а горизонтальная подалгебра $\mathfrak{D}_{\mathrm{H}}(\mathcal{M})$ имеет $\mathcal{A}$-базис $D=$ $\left\{D_{\sigma} \mid \sigma \in \mathbf{m}_{\mathcal{M}}\right\},\left[\mathbf{D}_{\sigma}, \mathbf{D}_{\tau}\right]=0$.

Следовательно, дуальный $\mathcal{A}$-модуль $\mathfrak{D}_{\mathrm{V}}^{*}(\mathcal{M})=\operatorname{Hom}_{\mathcal{A}}\left(\mathfrak{D}_{\mathrm{V}}(\mathcal{M}) ; \mathcal{A}\right)=\Omega_{\mathrm{V}}^{1}(\mathcal{M}, \mathcal{A})$ имеет дуальный базис $\mathcal{A}$-базис $\rho=\left\{\rho^{s} \mid s \in \mathbf{a}_{\mathcal{M}}\right\}, \rho^{s}\left(\partial_{t}\right)=\delta_{t}^{s}$. В частности, $\omega(X)=$ $\omega_{s} \rho^{s}\left(X^{t} \partial_{t}\right)=\omega_{s} X^{s}$ для всех $\omega \in \mathfrak{D}_{\mathrm{V}}^{*}(\mathcal{M}), X \in \mathfrak{D}_{\mathrm{V}}(\mathcal{M})$ и $\omega(X)=0$ для любых $\omega \in \mathfrak{D}_{\mathrm{V}}^{*}(\mathcal{M}), X \in \mathfrak{D}_{\mathrm{H}}(\mathcal{M})$.

\footnotetext{
2) Мы упрощаем обозначения для удобства записи.
} 
Дуальный $\mathcal{A}$-модуль $\mathfrak{D}_{\mathrm{H}}^{*}(\mathcal{M})=\operatorname{Hom}_{\mathcal{A}}\left(\mathfrak{D}_{\mathrm{H}}(\mathcal{M}) ; \mathcal{A}\right)=\Omega_{\mathrm{H}}^{1}(\mathcal{M}, \mathcal{A})$ имеет дуальный $\mathcal{A}$-базис $\boldsymbol{\vartheta}=\left\{\boldsymbol{\vartheta}^{\sigma} \mid \sigma \in \mathbf{m}_{\mathcal{M}}\right\}, \boldsymbol{\vartheta}^{\sigma}\left(\mathbf{D}_{\tau}\right)=\delta_{\tau}^{\sigma}$. В частности, $\omega(X)=\omega_{\sigma} \boldsymbol{\vartheta}^{\sigma}\left(X^{\tau} \mathbf{D}_{\tau}\right)=$ $\omega_{\sigma} X^{\sigma}$ для всех $\omega \in \mathfrak{D}_{\mathrm{H}}^{*}(\mathcal{M}), X \in \mathfrak{D}_{\mathrm{H}}(\mathcal{M})$ и $\omega(X)=0$ для любых $\omega \in \mathfrak{D}_{\mathrm{H}}(\mathcal{M})$, $X \in \mathfrak{D}_{\mathrm{V}}(\mathcal{M})$.

СоглАшениЕ 4. Ниже мы еще больше упрощаем обозначения: $\partial, D$ означают вертикальный и горизонтальный базисы в $\mathfrak{D}(\mathcal{U})$, а $\rho, \vartheta$ означают дуальные базисы.

ПРЕДЛОЖЕНИЕ 23. Представления

$$
\Omega^{p q}(\mathcal{U}, \mathcal{V})=\left\{\omega=\frac{1}{p ! q !} \omega_{a_{1} \ldots a_{p} \mu_{1} \ldots \mu_{q}} \cdot \rho^{a_{1}} \wedge \ldots \wedge \rho^{a_{p}} \wedge \vartheta^{\mu_{1}} \wedge \ldots \wedge \vartheta^{\mu_{q}}\right\}
$$

справедливы для всех $p \in \mathbb{Z}_{+} u 0 \leqslant q \leqslant m$, где коэфбиииенты $\omega_{a_{1} \ldots a_{p} \mu_{1} \ldots \mu_{q}} \in \mathcal{V}$ кососимметрические по индексам $a_{1}, \ldots, \mu_{q}$.

Внешнее дифференцирование $d: \Omega^{p q}(\mathcal{U}, \mathcal{V}) \rightarrow \Omega^{p+1, q}(\mathcal{U}, \mathcal{V}) \oplus_{\mathbb{F}} \Omega^{p, q+1}(\mathcal{U}, \mathcal{V})$ также разбивается на вертикальную и горизонтальную части, $d=d_{\mathrm{V}}+d_{\mathrm{H}}$, где

$$
d_{\mathrm{V}}: \Omega^{p q}(\mathcal{U}, \mathcal{V}) \rightarrow \Omega^{p+1 . q}(\mathcal{U}, \mathcal{V}), \quad d_{\mathrm{H}}: \Omega^{p q}(\mathcal{U}, \mathcal{V}) \rightarrow \Omega^{p, q+1}(\mathcal{U}, \mathcal{V})
$$

Действительно, согласно формуле Картана:

- $d v=\varkappa \partial_{a} v \cdot \rho^{a}+\varkappa D_{\mu} v \cdot \vartheta^{\mu}$ для всех $v \in \mathcal{V}$, следовательно, $d_{\mathrm{V}} v=\varkappa \partial_{a} v \cdot \rho^{a}, \quad d_{\mathrm{H}} v=\varkappa D_{\mu} v \cdot \vartheta^{\mu}$

- $d \rho^{a}=\Gamma_{\mu b}^{a} \cdot \rho^{b} \wedge \vartheta^{\mu}$ для всех $\rho^{a} \in \rho$, следовательно, $d_{\mathrm{V}} \rho^{a}=0, \quad d_{\mathrm{H}} \rho^{a}=\Gamma_{\mu b}^{a} \cdot \rho^{b} \wedge \vartheta^{\mu} ;$

- $d \vartheta^{\mu}=0$ для всех $\vartheta^{\mu} \in \vartheta$, следовательно, $d_{\mathrm{V}, \mathrm{H}} \vartheta^{\mu}=0$.

В силу предложений 15 и 23 эти формулы позволяют вычислить дифференциалы $d_{\mathrm{V}} \omega$ и $d_{\mathrm{H}} \omega$ для всякой формы $\omega \in \Omega^{p q}(\mathcal{U}, \mathcal{V})$.

ПреДЛОЖЕНИЕ 24. Равенство $d \circ d=0$ влечет равенства

$$
d_{\mathrm{V}} \circ d_{\mathrm{V}}=d_{\mathrm{V}} \circ d_{\mathrm{H}}+d_{\mathrm{H}} \circ d_{\mathrm{V}}=d_{\mathrm{H}} \circ d_{\mathrm{H}}=0 .
$$

ПРЕДЛОЖЕНИЕ 25. Определен вариационный бикомплекс

$$
\left\{\Omega^{p q}(\mathcal{U}, \mathcal{V}) ; d_{\mathrm{V}}^{p q}, d_{\mathrm{H}}^{p q} \mid p, q \in \mathbb{Z}\right\}, \quad \text { əde } \quad d_{\mathrm{V}, \mathrm{H}}^{p q}=\left.d_{\mathrm{V}, \mathrm{H}}\right|_{\Omega^{p q}(\mathcal{U}, \mathcal{V})}
$$

Вертикальные и горизонтальные когомологии этого бикомплекса определяются следующим образом:

$$
H_{\mathrm{V}}^{p q}(\mathcal{U}, \mathcal{V})=\operatorname{Ker} d_{\mathrm{V}}^{p q} / \operatorname{Im} d_{\mathrm{V}}^{p-1, q}, \quad H_{\mathrm{H}}^{p q}(\mathcal{U}, \mathcal{V})=\operatorname{Ker} d_{\mathrm{H}}^{p q} / \operatorname{Im} d_{\mathrm{H}}^{p, q-1}
$$

для всех $p, q \in \mathbb{Z}$.

Для сокращения обозначений ниже в этой секции мы опускаем аргументы $\mathcal{U}$ и $\mathcal{V}$ и пишем $\Omega^{p q}$ вместо $\Omega^{p q}(\mathcal{U}, \mathcal{V}), H_{\mathrm{V}, \mathrm{H}}^{p q}$ вместо $H_{\mathrm{V}, \mathrm{H}}^{p q}(\mathcal{U}, \mathcal{V}), \mathfrak{D}$ вместо $\mathfrak{D}(\mathcal{U})$ и т. д. 
Для любых $p, q \in \mathbb{Z}$ определены вторые дифференциалы:

- $d_{\mathrm{HV}}^{p q} \in \operatorname{Hom}_{\mathbb{F}}\left(H_{\mathrm{V}}^{p q}, H_{\mathrm{V}}^{p, q+1}\right), \quad \omega_{\mathrm{V}}^{p q} \mapsto d_{\mathrm{HV}}^{p q} \omega_{\mathrm{V}}^{p q}=\left[d_{\mathrm{H}} \omega^{p q}\right]_{\mathrm{V}}$,

- $d_{\mathrm{VH}}^{p q} \in \operatorname{Hom}_{\mathbb{F}}\left(H_{\mathrm{H}}^{p q}, H_{\mathrm{H}}^{p+1, q}\right), \quad \omega_{\mathrm{H}}^{p q} \mapsto d_{\mathrm{VH}}^{p q} \omega_{\mathrm{H}}^{p q}=\left[d_{\mathrm{V}} \omega^{p q}\right]_{\mathrm{H}}$,

где

- $\omega_{\mathrm{V}}^{p q}=\left[\omega^{p q}\right]_{\mathrm{V}}=\omega^{p q}+d_{\mathrm{V}} \Omega^{p-1, q} \in H_{\mathrm{V}}^{p q}, \omega^{p q} \in \Omega^{p q}, d_{\mathrm{V}} \omega^{p q}=0$,

- $\omega_{\mathrm{H}}^{p q}=\left[\omega^{p q}\right]_{\mathrm{H}}=\omega^{p q}+d_{\mathrm{H}} \Omega^{p, q-1} \in H_{\mathrm{H}}^{p q}, \omega^{p q} \in \Omega^{p q}, \quad d_{\mathrm{H}} \omega^{p q}=0$.

Следовательно, определены комплексы

$$
\left\{H_{\mathrm{V}}^{p q} ; d_{\mathrm{HV}}^{p q} \mid q \in \mathbb{Z}\right\}, \quad p \in \mathbb{Z}, \quad\left\{H_{\mathrm{H}}^{p q} ; d_{\mathrm{VH}}^{p q} \mid p \in \mathbb{Z}\right\}, \quad q \in \mathbb{Z},
$$

с пространствами вторых когомологий:

$$
\begin{aligned}
H_{\mathrm{HV}}^{p q} & =\operatorname{Ker} d_{\mathrm{HV}}^{p q} / \operatorname{Im} d_{\mathrm{HV}}^{p, q-1}= \\
& =\left\{\omega_{\mathrm{HV}}^{p q}=\omega^{p q}+d_{\mathrm{V}} \Omega^{p-1, q} \mid \omega^{p q} \in \Omega^{p q}, d_{\mathrm{V}} \omega^{p q}=0, d_{\mathrm{H}} \omega^{p q} \in d_{\mathrm{V}} \Omega^{p-1, q+1}\right\}, \\
H_{\mathrm{VH}}^{p q} & =\operatorname{Ker} d_{\mathrm{VH}}^{p q} / \operatorname{Im} d_{\mathrm{VH}}^{p-1, q}= \\
& =\left\{\omega_{\mathrm{VH}}^{p q}=\omega^{p q}+d_{\mathrm{H}} \Omega^{p, q-1} \mid \omega^{p q} \in \Omega^{p q}, d_{\mathrm{H}} \omega^{p q}=0, d_{\mathrm{V}} \omega^{p q} \in d_{\mathrm{H}} \Omega^{p+1, q-1}\right\} .
\end{aligned}
$$

Все дальнейшие дифференциалы и когомологии тривиальные.

ТЕорема 1. В регулярном случае элементы спектральной последовательности $\left\{E_{r}^{p q}, d_{r}^{p q}\right\}$ имеют вид

- $E_{0}^{p q}=\Omega_{p}^{p+q} / \Omega_{p+1}^{p+q}=\Omega^{p q}, d_{0}^{p q}=d_{\mathrm{H}}^{p q}: \Omega^{p q} \rightarrow \Omega^{p, q+1}$;

- $E_{1}^{p q}=\operatorname{Ker} d_{\mathrm{H}}^{p q} / \operatorname{Im} d_{\mathrm{H}}^{p, q-1}=H_{\mathrm{H}}^{p q}, d_{1}^{p q}=d_{\mathrm{VH}}^{p q}: H_{\mathrm{H}}^{p q} \rightarrow H_{\mathrm{H}}^{p+1, q}$;

- $E_{2}^{p q}=\operatorname{Ker} d_{\mathrm{VH}}^{p q} / \operatorname{Im} d_{\mathrm{VH}}^{p-1, q}=H_{\mathrm{VH}}^{p q}, d_{2}^{p q}=0$;

- $E_{r}^{p q}=E_{2}^{p q}=H_{\mathrm{VH}}^{p q}, d_{r}^{p q}=0, r \geqslant 2$;

- $\lim _{r \rightarrow \infty} E_{r}^{p q}=E_{2}^{p q}=E_{\infty}^{p q}$.

ДокАЗАТЕЛЬство теоремы 1 базируется на общих свойствах спектральных последовательностей и приведенных выше вычислениях для вариационного бикомплекса.

Пусть $X=X_{\mathrm{V}}+X_{\mathrm{H}}=X^{a} \partial_{a}+X^{\mu} D_{\mu} \in \mathfrak{D}=\mathfrak{D}_{\mathrm{V}}+\mathfrak{D}_{\mathrm{H}}$. Тогда справедливы следующие утверждения:

- $i_{X} F=0$ для любого $F \in \mathcal{V}$;

- $i_{X} \rho^{a}=X^{a}=i_{X_{\mathrm{V}}} \rho^{a}$ для любого $a \in \mathbf{a}$;

- $i_{X} \vartheta^{\mu}=X^{\mu}=i_{X_{\mathrm{H}}} \vartheta^{\mu}$ для любого $\mu \in \mathbf{m}$;

- $L_{X} F=(\varkappa X) F$ для любого $F \in \mathcal{V}$;

- $L_{X} \rho^{a}=\left(\partial_{b} X^{a}-\Gamma_{\mu b}^{a} X^{\mu}\right) \cdot \rho^{b}+\left(\nabla_{\mu} X_{\mathrm{V}}\right)^{a} \cdot \vartheta^{\mu}$ для любого $a \in \mathbf{a}$;

- $L_{X} \vartheta^{\mu}=\partial_{a} X^{\mu} \cdot \rho^{a}+D_{\nu} X^{\mu} \cdot \vartheta^{\nu}$ для любого $\mu \in \mathbf{m}$.

Напомним, что $\left(\nabla_{\mu} X\right)^{a}=D_{\mu} X^{a}+\Gamma_{\mu b}^{a} X^{b}=\left(\nabla_{\mu} X_{\mathrm{V}}\right)^{a}$ (см. определение 7 ). В силу предложений 12 и 23 эти формулы позволяют вычислить формы $i_{X} \omega$ и $L_{X} \omega$ для любой формы $\omega \in \Omega^{p q}$. 
ПРЕДЛОЖЕНИЕ 26. Пусть $\omega \in \Omega^{p q}, p, q \in \mathbb{Z}, X \in \mathfrak{D}$, тогда

$$
i_{X} \omega \in\left\{\begin{array} { l l } 
{ \Omega ^ { p - 1 , q } , } & { X \in \mathfrak { D } _ { \mathrm { V } } , } \\
{ \Omega ^ { p , q - 1 } , } & { X \in \mathfrak { D } _ { \mathrm { H } } , }
\end{array} \quad L _ { X } \omega \in \left\{\begin{array}{ll}
\Omega^{p+1, q-1} \oplus \Omega^{p q} \oplus \Omega^{p-1, q+1}, & X \in \mathfrak{D}, \\
\Omega^{p q}, & X \in \mathcal{E}
\end{array}\right.\right.
$$

Напомним, что $\mathcal{E} \subset \mathfrak{D}($ см. предложения 7 и 11).

ПреДЛОЖЕНИЕ 27. Для любъх $X \in \mathcal{E}, p, q \in \mathbb{Z}$, определены эндоморбизмы

$$
\left[L_{X}\right]^{p q} \in \operatorname{End}_{\mathbb{F}}\left(H_{\mathrm{V}, \mathrm{H}}^{p q}\right), \quad\left[\omega^{p q}\right]_{\mathrm{V}, \mathrm{H}} \mapsto\left[L_{X}\right]^{p q}\left[\omega^{p q}\right]_{\mathrm{V}, \mathrm{H}}=\left[L_{X} \omega^{p q}\right]_{\mathrm{V}, \mathrm{H}},
$$

где $\left[\omega^{p q}\right]_{\mathrm{V}}=\omega^{p q}+d_{\mathrm{V}}^{p-1, q} \Omega^{p-1, q},\left[\omega^{p q}\right]_{\mathrm{H}}=\omega^{p q}+d_{\mathrm{H}}^{p, q-1} \Omega^{p, q-1}$.

\section{6. ДИФФЕРЕНЦИАЛЬНЫЕ АЛГЕБРЫ В УРАВНЕНИЯХ В ЧАСТНЫХ ПРОИЗВОДНЫХ}

6.1. Обозначения. Введем следующие обозначения:

- $\mathrm{X}=\mathbb{R}^{\mathbf{m}}=\left\{x=\left(x^{\mu}\right) \mid x^{\mu} \in \mathbb{R}, \mu \in \mathbf{m}\right\}$ есть линейное пространство независимых переменных;

- $\mathrm{U}=\mathbb{R}^{\mathrm{A}}=\left\{u=\left(u^{\alpha}\right) \mid u^{\alpha} \in \mathbb{R}, \alpha \in \mathrm{A}\right\}$ есть линейное пространство зависимых переменных, где А - конечное множество индексов;

- $\mathbf{U}=\mathbb{R}_{\mathrm{I}}^{\mathrm{A}}=\left\{\mathbf{u}=\left(u_{i}^{\alpha}\right) \mid u_{i}^{a} \in \mathbb{R}, \alpha \in \mathrm{A}, i \in \mathrm{I}\right\}$ есть линейное пространство дифференциальньх переменных, $\mathrm{I}=\mathbb{Z}_{+}^{\mathbf{m}}$ (заметим, $\operatorname{dim} \mathbf{U}=\infty$ );

- $\mathcal{A}=\mathcal{C}_{\text {fin }}^{\infty}(\mathrm{XU})$ есть унитальная ассоциативная коммутативная алгебра $\mathbb{F}$-значных гладких функций, зависящих от конечного числа аргументов $x^{\mu}, u_{i}^{\alpha}$, $\mathrm{XU}=\mathrm{X} \times \mathbf{U}$.

В этом случае $\mathfrak{M}(\mathcal{A})=\mathcal{A}$, поскольку алгебра $\mathcal{A}$ унитальная. $\mathcal{A}$-алгебра Ли $\mathfrak{D}=\mathfrak{D}(\mathcal{A})$ имеет стандартный $\mathcal{A}$-базис $\left\{\partial_{u_{i}^{\alpha}}, \partial_{x^{\mu}} \mid \alpha \in \mathrm{A}, i \in \mathrm{I}, \mu \in \mathbf{m}\right\}$, где $\partial_{u_{i}^{\alpha}}, \partial_{x^{\mu}}$ суть частные производные.

В алгебраическом подходе к уравнениям в частных производных $\mathcal{A}$-алгебра Ли $\mathfrak{D}$ разбивается как $\mathfrak{D}=\mathfrak{D}_{\mathrm{V}} \oplus_{\mathcal{A}} \mathfrak{D}_{\mathrm{H}}$, где

- вертикальная подалгебра $\mathfrak{D}_{\mathrm{V}}$ имеет $\mathcal{A}$-базис $\partial=\left\{\partial_{u_{i}^{\alpha}} \mid \alpha \in \mathrm{A}, i \in \mathrm{I}\right\}$, $\left[\partial_{u_{i}^{\alpha}}, \partial_{u_{j}^{\beta}}\right]=0$

- горизонтальная подалгебра $\mathfrak{D}_{\mathrm{H}}$ имеет $\mathcal{A}$-базис $D=\left\{D_{\mu} \mid \mu \in \mathbf{m}\right\}, \quad D_{\mu}=$ $\partial_{x^{\mu}}+u_{i+(\mu)}^{\alpha} \partial_{u_{i}^{\alpha}}, i+(\mu)=\left(i^{1}, \ldots, i^{\mu}+1, \ldots, i^{m}\right), \quad\left[D_{\mu}, D_{\nu}\right]=0$.

Горизонтальные базисные дифференцирования $D_{\mu}$ называются полными производными, они характеризуются правилом дифференцирования сложной функиии:

$$
\partial_{x^{\mu}}\left(\left.f(x, \mathbf{u})\right|_{\mathbf{u}=\phi(x)}\right)=\left.\left(D_{\mu} f(x, \mathbf{u})\right)\right|_{\mathbf{u}=\phi(x)}
$$

для всех $\mu \in \mathbf{m}, f \in \mathcal{A}$ и $\phi \in \mathcal{C}^{\infty}(\mathrm{X} ; \mathrm{U})$, где $\phi(x)=\left(\phi^{\alpha}(x)\right), \phi(x)=\left(\phi_{i}^{\alpha}(x)\right), \phi_{i}^{\alpha}(x)=$ $\partial_{x^{i}} \phi^{\alpha}(x), \partial_{x^{i}}=\left(\partial_{x^{1}}\right)^{i^{1}} \ldots\left(\partial_{x^{m}}\right)^{i^{m}}, i=\left(i^{1}, \ldots, i^{m}\right) \in \mathrm{I}$.

- Коммутаторы $\left[D_{\mu}, \partial_{u_{i}^{\alpha}}\right]=-\partial_{u_{i-(\mu)}^{\alpha}}$, так что связность $\Gamma=\left(\Gamma_{\mu \alpha j}^{i \beta}\right), \Gamma_{\mu \alpha j}^{i \beta}=$ $-\delta_{\alpha}^{\beta} \delta_{j+(\mu)}^{i}, \mu \in \mathbf{m}, \alpha, \beta \in \mathrm{A}, i, j \in \mathrm{I}$.

Итак, определена регулярная унитальная дифференциальная алгебра $\left(\mathcal{A}, \mathfrak{D}_{\mathrm{H}}\right)$. 
Здесь коммутатор $\left[D_{\mu}, X\right]=\left(\nabla_{\mu} \zeta\right)_{i}^{\alpha} \partial_{u_{i}^{\alpha}}$ для любого $X=\zeta_{i}^{\alpha} \partial_{u_{i}^{\alpha}} \in \mathfrak{D}_{\mathrm{V}}$, где

$$
\nabla_{\mu} \in \operatorname{End}_{\mathbb{F}}\left(\mathcal{A}_{\mathrm{I}}^{\mathrm{A}}\right), \quad \zeta=\left(\zeta_{i}^{\alpha}\right) \mapsto \nabla_{\mu} \zeta=\left(\left(\nabla_{\mu} \zeta\right)_{i}^{\alpha}\right), \quad\left(\nabla_{\mu} \zeta\right)_{i}^{\alpha}=D_{\mu} \zeta_{i}^{\alpha}-\zeta_{i+(\mu)}^{\alpha} .
$$

ПРЕДЛОЖЕНИЕ 28. Коммутатор $\left[\nabla_{\mu}, \nabla_{\nu}\right]=0$ для всех $\mu, \nu \in \mathbf{m}$, m.е. кривизна $F=\left(F_{\mu \nu}\right)=0$.

ОПРЕДЕЛЕНИЕ 19. Число $n \in \mathbb{Z}_{+}$называется порядком дифференииалъной функиии $f \in \mathcal{A}$, если $\partial_{u_{i}^{\alpha}} f \neq 0$ для некоторых $\alpha \in \mathrm{A}$ и $i \in \mathrm{I},|i|=i^{1}+\cdots+i^{m}=n$, тогда как $\partial_{u_{j}^{\beta}} f=0$ для всех $\beta \in \mathrm{A}$ и $j \in \mathrm{I},|j|=j^{1}+\cdots+j^{m}>n$.

Лемма 1. Подалгебра $\mathcal{A}_{\mathcal{D}}=\mathcal{A}_{\mathrm{H}}^{(0)}=\mathbb{F}$.

ДокАзАТЕЛьство леммы 1 опирается на конечность порядка дифференциальных функций.

ПрЕДЛОЖЕНИЕ 29. В филътрации $\left\{\mathcal{A}_{\mathrm{H}}^{(q)} \mid q \in \mathbb{Z}_{+}\right\}$линейнъе пространства

$$
\mathcal{A}_{\mathrm{H}}^{(q)}=\mathbb{F}_{q}[x]=\left\{f(x)=\sum_{|i| \leqslant q} f_{i} x^{i} \mid f_{i} \in \mathbb{F}\right\}
$$

являются пространствами многочленов q-го порядка по переменной $x \in \mathrm{X}$, где $x^{i}=\left(x^{1}\right)^{i^{1}} \ldots\left(x^{m}\right)^{i^{m}}, i \in \mathrm{I},|i|=i^{1}+\cdots+i^{m}$.

Предел $\lim _{q \rightarrow \infty} \mathcal{A}_{\mathrm{H}}^{(q)}$ зависит от выбранной топологии. Так, если выбрать естественную топологию линейного пространства всех многочленов $\mathbb{F}[x]$, получим $\lim _{q \rightarrow \infty} \mathcal{A}_{\mathrm{H}}^{(q)}=\mathbb{F}[x]$, а если выбрать естественную топологию линейного пространства всех гладких функций $\mathcal{C}^{\infty}(\mathrm{X} ; \mathbb{F})$, получим $\lim _{q \rightarrow \infty} \mathcal{A}_{\mathrm{H}}^{(q)}=\mathcal{C}^{\infty}(\mathrm{X} ; \mathbb{F})$.

Лемма 2. Справедливы равенства

$$
\left(\nabla_{r} \zeta\right)_{i}^{\alpha}=\sum_{k+j=r}(-1)^{k}\left(\begin{array}{l}
r \\
k
\end{array}\right) D_{j} \zeta_{i+k}^{\alpha}
$$

где $\alpha \in \mathrm{I}, \quad i, r, k, j \in \mathrm{I}, \quad \nabla_{r}=\left(\nabla_{1}\right)^{r^{1}} \ldots\left(\nabla_{m}\right)^{r^{m}}, D_{j}=\left(D_{1}\right)^{j^{1}} \ldots\left(D_{m}\right)^{j^{m}}$.

Для доказательства леммы 2 следует воспользоваться предложением 29, индукцией по $r$ и хорошо известным равенством $\left(\begin{array}{l}r \\ k\end{array}\right)+\left(\begin{array}{c}r \\ k-(\mu)\end{array}\right)=\left(\begin{array}{c}r+(\mu) \\ k\end{array}\right)$.

ЗАмЕчАниЕ 1. Мы используем стандартные мультииндексные обозначения, в частности $(-1)^{r}=(-1)^{|r|}=(-1)^{r^{1}+\cdots+r^{m}},\left(\begin{array}{l}r \\ k\end{array}\right)=\left(\begin{array}{l}r^{1} \\ k^{1}\end{array}\right) \ldots\left(\begin{array}{l}r^{m} \\ k^{m}\end{array}\right)$.

ОПРЕДЕЛЕНИЕ 20. Для каждого $k \in \mathrm{I}$ определим линейное подпространство

$$
\Phi^{k}=\left\{\epsilon_{\phi}^{k}=\left(\epsilon_{\phi i}^{k \alpha}\right) \in \mathcal{A}_{\mathrm{I}}^{\mathrm{A}} \mid \epsilon_{\phi i}^{k \alpha}=\left(\begin{array}{c}
i \\
k
\end{array}\right) D_{i-k} \phi^{\alpha}, \phi=\left(\phi^{\alpha}\right) \in \mathcal{A}^{\mathrm{A}}\right\} \subset \mathcal{A}_{\mathrm{I}}^{\mathrm{A}} .
$$

Положим также $\Phi^{k}=0$ при $k \notin \mathrm{I}$.

ЛЕмма 3. Для любъх $r, k \in \mathrm{I}$ отображение

$$
\nabla_{r} \in \operatorname{Hom}_{\mathbb{F}}\left(\Phi^{k} ; \Phi^{k-r}\right), \quad \epsilon_{\phi}^{k} \mapsto \nabla_{r} \epsilon_{\phi}^{k}=(-1)^{r} \epsilon_{\phi}^{k-r} .
$$

В частности, $\nabla_{r} \epsilon_{\phi}^{k}=0$ для всех $k-r \notin \mathrm{I} u \phi \in \mathcal{A}^{\mathrm{A}}$. 
ДокАзАТЕЛЬСтво. Действительно,

$$
\begin{aligned}
\left(\nabla_{\mu} \epsilon_{\phi}^{k}\right)_{i}^{\alpha} & =D_{\mu} \epsilon_{\phi i}^{k \alpha}-\epsilon_{\phi, i+(\mu)}^{k \alpha}=\left(\begin{array}{c}
i \\
k
\end{array}\right) D_{i+(\mu)-k} \phi^{\alpha}-\left(\begin{array}{c}
i+(\mu) \\
k
\end{array}\right) D_{i+(\mu)-k} \phi^{\alpha}= \\
& =\left(\left(\begin{array}{c}
i \\
k
\end{array}\right)-\left(\begin{array}{c}
i+(\mu) \\
k
\end{array}\right)\right) D_{i+(\mu-k)} \phi^{\alpha}=-\left(\begin{array}{c}
i \\
k-(\mu)
\end{array}\right) D_{i-k+(\mu) \phi}=-\epsilon_{\phi i}^{k-(\mu), \alpha} .
\end{aligned}
$$

Для завершения доказательства следует воспользоваться индукцией по индексу $r$.

Теорема 2. Для каждого $\zeta=\left(\zeta_{i}^{\alpha}\right) \in \mathcal{A}_{\mathrm{I}}^{\mathrm{A}}$ имеется единственное представление

$$
\zeta=\sum_{k \in \mathrm{I}} \epsilon_{\phi_{k}}^{k}, \quad \phi_{k}=\left(\phi_{k}^{\alpha}\right) \in \mathcal{A}^{\mathrm{A}}, \quad \phi_{k}^{\alpha}=\sum_{i+j=k}(-1)^{j}\left(\begin{array}{c}
k \\
i
\end{array}\right) D_{j} \zeta_{i}^{\alpha} .
$$

Другими словами, линейное пространство $\mathcal{A}_{\mathrm{I}}^{\mathrm{A}}$ I-градуировано линейными пространствами $\Phi^{k}$, m.e. $\mathcal{A}_{\mathrm{I}}^{\mathrm{A}}=\oplus_{k \in \mathrm{I}} \Phi^{k}$.

ДоКАЗАТЕЛЬСТво. Действительно, для данного $\zeta \in \mathcal{A}_{\mathrm{I}}^{\mathrm{A}}$ необходимо найти функции $\phi_{k} \in \mathcal{A}^{\mathrm{A}}, k \in \mathrm{I}$, такие, чтобы выполнялось равенство $\zeta=\sum_{k \in \mathrm{I}} \epsilon_{\phi_{k}}^{k}$. Применяя к обеим частям этого равенства операторы $\nabla_{r}, r \in \mathrm{I}$, получим (см. леммы 2,3 )

$$
\begin{aligned}
\left(\nabla_{r} \zeta\right)_{i}^{\alpha} & =\sum_{k+j=r}(-1)^{k}\left(\begin{array}{l}
r \\
k
\end{array}\right) D_{j} \zeta_{i+k}^{\alpha}=\sum_{k \in \mathrm{I}}\left(\nabla_{r} \epsilon_{\phi_{k}}^{k}\right)_{i}^{\alpha}=(-1)^{r} \sum_{k \in \mathrm{I}}\left(\epsilon_{\phi_{k}}^{k-r}\right)_{i}^{\alpha}= \\
& =(-1)^{r} \sum_{j \in \mathrm{I}}\left(\epsilon_{\phi_{j+r}^{j}}^{j}\right)=(-1)^{r} \sum_{j \in \mathrm{I}}\left(\begin{array}{l}
i \\
j
\end{array}\right) D_{i-j} \phi_{i+r}^{\alpha} .
\end{aligned}
$$

Таким образом, следует удовлетворить равенствам

$$
\sum_{j \in \mathrm{I}}\left(\begin{array}{l}
i \\
j
\end{array}\right) D_{i-j} \phi_{i+r}^{\alpha}=\sum_{k+j=r}(-1)^{k+r}\left(\begin{array}{l}
r \\
k
\end{array}\right) D_{j} \zeta_{i+k}^{\alpha}, \quad \alpha \in \mathrm{A}, \quad i \in \mathrm{I} .
$$

В частности, для $i=0$ имеем

$$
\phi_{r}^{\alpha}=\sum_{k+j=r}(-1)^{k+r}\left(\begin{array}{l}
r \\
k
\end{array}\right) D_{j} \zeta_{k}^{\alpha} .
$$

Простая проверка показывает, что этот единственный выбор решает задачу.

СлЕДСтвиЕ 1. Справедливы следующие утверждения:

- В фильтрачии $\left\{\mathcal{E}^{(q)} \mid q \in \mathbb{Z}_{+}\right\}$линейные пространства $\mathcal{E}^{(q)}=\bigoplus_{|k| \leqslant q} \mathcal{E}^{k}, \quad \mathcal{E}^{k}=\left\{X=\epsilon_{\phi i}^{k \alpha} \cdot \partial_{u_{i}^{\alpha}} \mid \epsilon_{\phi}^{k}=\left(\epsilon_{\phi i}^{k \alpha}\right) \in \Phi^{k}\right\} ;$

- $\lim _{q \rightarrow \infty} \mathcal{E}^{(q)}=\mathfrak{D}_{\mathrm{V}}=\bigoplus_{k \in \mathrm{I}} \mathcal{E}^{k}$.

Рассмотрим вариационный бикомплекс

$$
\left\{\Omega^{p q} ; d_{\mathrm{V}}^{p q}, d_{\mathrm{H}}^{p q} \mid p \in \mathbb{Z}_{+}, 0 \leqslant q \leqslant m\right\}, \quad \text { где } \quad \Omega^{p q}=\Omega^{p q}(\mathcal{A}, \mathcal{A}) .
$$

Здесь 


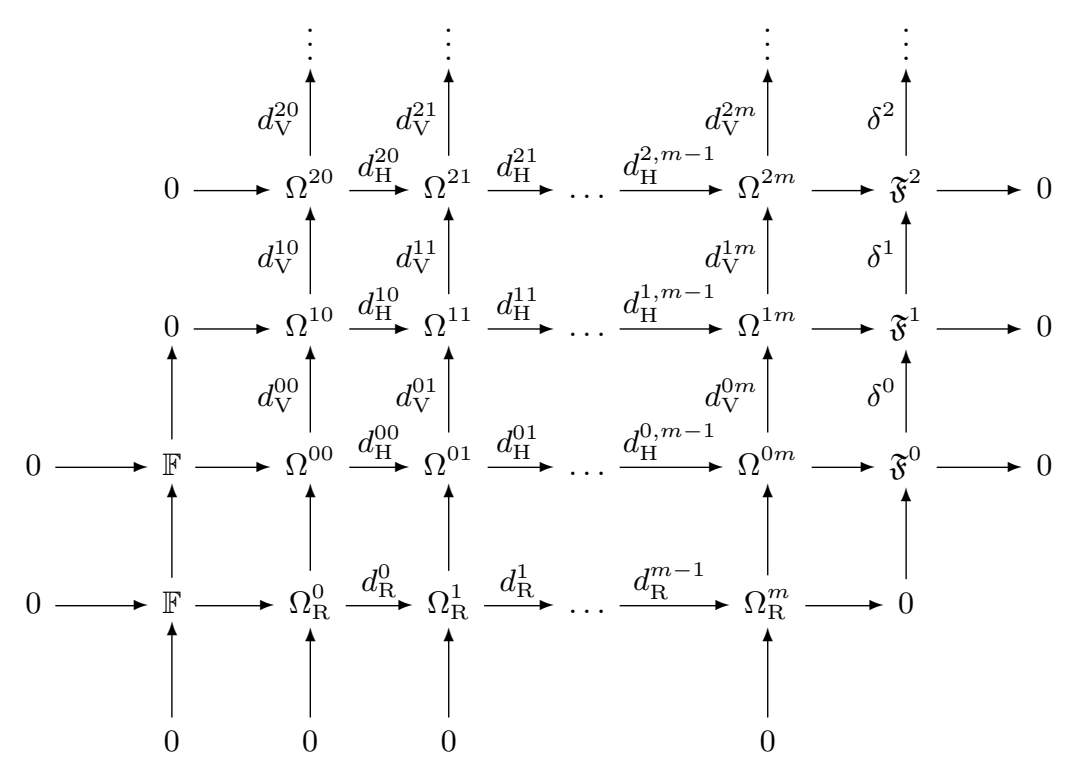

Рис. 1

- вертикальный $\mathcal{A}$-базис $\partial=\left\{\partial_{u_{i}^{\alpha}} \mid \alpha \in \mathrm{A}, i \in \mathrm{I}\right\}$ имеет дуальный базис $\rho=$ $\left\{\rho_{i}^{\alpha}=d u_{i}^{\alpha}-u_{i+(\mu)}^{\alpha} d x^{\mu} \mid \alpha \in \mathrm{A}, i \in \mathrm{I}\right\}, \quad \rho_{i}^{\alpha}\left(\partial_{u_{j}^{\beta}}\right)=\delta_{\beta}^{\alpha} \delta_{i}^{j}, \quad \rho_{i}^{\alpha}\left(D_{\mu}\right)=0$,

- горизонтальный $\mathcal{A}$-базис $D=\left\{D_{\mu} \mid \mu \in \mathbf{m}\right\}$ имеет дуальный базис $\vartheta=\left\{\vartheta^{\mu}=\right.$ $\left.d x^{\mu} \mid \mu \in \mathbf{m}\right\}, d x^{\mu}\left(\partial_{u_{i}^{\alpha}}\right)=0, d x^{\mu}\left(D_{\nu}\right)=\delta_{\nu}^{\mu}$.

Пополним вариационный бикомплекс, добавив

- горизонтальный комплекс $\left\{\Omega_{\mathrm{R}}^{q} ; d_{\mathrm{R}}^{q} \mid 0 \leqslant q \leqslant m\right\}$, где $\Omega_{\mathrm{R}}^{q}=\Omega^{q}\left(\mathcal{C}^{\infty}(X)\right)$, $d_{\mathrm{R}}^{q}=d^{q}$, т. е. стандартный комплекс де Рама пространства $X=\mathbb{R}^{\mathbf{m}}$;

- вертикальный комплекс $\left\{\mathfrak{F}^{p} ; \delta^{p} \mid p \in \mathbb{Z}_{+}\right\}$, где $\mathfrak{F}^{p}=\Omega^{p m} / \operatorname{Im} d^{p, m-1}$ суть линейные факторпространства функциональных $p$-форм, $\delta^{p}$ суть линейные фактор-дифференциалы, $[\omega] \mapsto \delta^{p}[\omega]=\left[d_{\mathrm{V}}^{p m} \omega\right],[\omega]$ есть класс эквивалентности формы $\omega \in \Omega^{p m}$.

Полученный пополненный бикомплекс приведен на рис. 1.

ТеОрема 3. Пополненный бикомплекс ациклический, m. е. все его строки и столбиљи точные.

Подробное доказательство этой знаменитой теоремы, ее историю и связанные с ней результаты можно найти, например, в [6], [9], [10].

В алгебраическом подходе нелинейная система уравнений в частных производных записывается в виде $F=0$, где $F=\left\{F^{\sigma} \in \mathcal{A} \mid \sigma \in \mathrm{S}\right\}, \mathrm{S}$ - множество индексов. $\mathrm{C}$ ней ассоциируется дифференциальный идеал $\mathcal{I}_{F}=\left\{f=P_{\sigma}(\mathcal{D}) F^{\sigma} \mid P_{\sigma}(\mathcal{D}) \in\right.$ $\mathcal{A}[\mathcal{D}], \sigma \in \mathrm{S}\}$, где $\mathcal{A}[\mathcal{D}]$ - унитальная ассоциативная некоммутативная алгебра всех многочленов от $\mathcal{D}=\left\{D_{\mu} \mid \mu \in \mathbf{m}\right\}$ с коэффициентами в $\mathcal{A}$. Дифференциальная фактор-алгебра $(\mathcal{A}, \mathcal{D})$ называется дифференциальной алгеброй, ассоииированной $c$ системой $F=0$ (подробнее см., например, [15], [16]). Такая конструкция позволяет выписать спектральную последовательность (спектральную последовательность 
Виноградова [8]), ассоциированную с системой $F=0$. Вычисление этой последовательности или ее отдельных членов совершенно другая задача, обычно чрезвычайно трудная. Для построения ассоциированного вариационного бикомплекса сначала следует записать ассоциированную дифференциальную алгебру $(\mathcal{A}, \mathcal{D})$ в регулярной форме, а затем следовать процедуре, изложенной в разделе 5. По-прежнему остается задача вычисления всего бикомплекса или отдельных его членов.

\section{7. ЗАКЛЮЧЕНИЕ}

Техника и методы, изложенные выше, были использованы в работах автора [11]-[15], [17], [18]. Они могут оказаться полезными в исследованиях [19]-[26].

\section{Список литературы}

[1] J.F. Ritt, Differential Algebra, American Mathematical Society Colloquium Publications, 33, AMS, New York, 1950.

[2] E. R. Kolchin, Differential Algebra and Algebraic Groups, Acad. Press, New York, 1973.

[3] I. Kaplansky, An Introduction to Differential Algebra, Hermann, Paris, 1957.

[4] С. Маклейн, Гомология, М., Мир, 1966.

[5] Р. Годеман, Алгебраическая топология и теория пучков, ИЛ, М., 1961.

[6] П. Олвер, Приложения групп Ли к дифференииальным уравнениям, Мир, М., 1989.

[7] V.V. Zharinov, Lecture Notes on Geometrical Aspects of Partial Differential Equations, Series on Soviet and East European Mathematics, 9, World Sci., Singapore-New Jersey-London-Hong Kong, 1992.

[8] А. М. Виноградов, "Одна спектральная последовательность, связанная с нелинейным дифференциальным уравнением, алгебро-геометрические основания лагранжевой теории поля со связями", Докл. АН СССР, 238:5 (1978), 1028-1031.

[9] T. Tsujishita, "On variation bicomplexes associated to differential equations", Osaka J. Math., 19:2 (1982), 311-363.

[10] I. M. Anderson, The variational bicomplex, Dept. Math., Utah State University, Logan, UT, 2004.

[11] V.V. Zharinov, "On differentiations in differential algebras", Integral Transform. Spec. Funct., 4:1-2 (1996), 163-180.

[12] В. В. Жаринов, “Формальный комплекс де Рама", ТМФ, 174:2 (2013), 256-271.

[13] В.В. Жаринов, “Алгебраический взгляд на калибровочные теории”, ТМФ, 180:2 (2014), 217-233.

[14] В. В. Жаринов, “Анализ в алгебрах и модулях", Тр. МИАН, 301 (2018), в печати.

[15] В. В. Жаринов, “О преобразовании Беклунда", ТМФ, 189:3 (2016), 323-334.

[16] В.В. Жаринов, “Алгебро-геометрические основы математической физики”, Леки. курсъ НОЦ, 9 (2008), 3-209.

[17] В. В. Жаринов, “Законы сохранения, дифференциальные тождества и связи уравнений в частных производных", ТМФ, 185:2 (2015), 227-251.

[18] В. В. Жаринов, "О гамильтоновых операторах в дифференциальных алгебрах", ТМФ, 193:3 (2017), 369-380.

[19] S. V. Kozyrev, A. A. Mironov, A.E. Teretenkov, I. V. Volovich, "Flows in nonequilibrium quantum systems and quantum photosynthesis", Infin. Dimens. Anal. Quantum Probab. Relat. Top., 20:4 (2017), 1750021, 19 pp., arXiv: 1612.00213.

[20] N. G. Marchuk, D. S. Shirokov, "General solutions of one class of field equations", Rep. Math. Phys., 78:3 (2016), 305-326, arXiv: 1406.6665. 
[21] М. О. Катанаев, "Векторные поля Киллинга и однородная и изотропная вселенная", УФН, 186:7 (2016), 763-775.

[22] А.Г. Сергеев, "Спинорная геометрия Дирака и некоммутативная геометрия Конна", Тр. МИАН, 298 (2017), 276-314.

[23] A. S. Trushechkin, I. V. Volovich, "Perturbative treatment of inter-site couplings in the local description of open quantum networks", Europhys. Lett., 113:3 (2016), 30005, 6 pp., arXiv: 1509.05754 .

[24] А.К. Гущин, "О разрешимости задачи Дирихле для неоднородного эллиптического уравнения второго порядка", Матем. сб., 206:10 (2015), 71-102.

[25] Ю.Н. Дрожжинов, "Многомерные тауберовы теоремы для обобщенных функций", УМН, 71:6(432) (2016), 99-154.

[26] И.В. Волович, С. В. Козырев, "Манипуляция состояниями вырожденной квантовой системы", Тр. МИАН, 294 (2016), 256-267.

Поступила в редакцию 10.03.2018 